\title{
Mortalidade de adolescentes e adultos jovens brasileiros entre 1990 e 2019: uma análise do estudo Carga Global de Doença
}

\author{
Mortality among Brazilian adolescents and young adults between \\ 1990 to 2019: an analysis of the Global Burden of Disease study
}

Deborah Carvalho Malta (http://orcid.org/0000-0002-8214-5734) ${ }^{1}$

Maria Cecília de Souza Minayo (https://orcid.org/0000-0001-6187-9301) ${ }^{2}$

Laís Santos de Magalhães Cardoso (http://orcid.org/0000-0002-1114-5470) ${ }^{3}$

Guilherme Augusto Veloso (https://orcid.org/0000-0002-5348-3793) ${ }^{4}$

Renato Azeredo Teixeira (http://orcid.org/0000-0002-1259-6812) 5,6

Isabella Vitral Pinto (https://orcid.org/0000-0002-3535-7208) 5,7

Mohsen Naghavi (https://orcid.org/0000-0003-3691-1458) ${ }^{8}$

\footnotetext{
${ }^{1}$ Departamento de

Enfermagem Materno-Infantil

e Saúde Pública, Escola de

Enfermagem, Universidade

Federal de Minas Gerais. Av.

Alfredo Balena 190, Santa

Efigênia. 30130-100 Belo

Horizonte MG Brasil.

dcmalta@uol.com.br

${ }^{2}$ Departamento de Estudos

sobre Violência e Saúde Jorge

Careli, Escola Nacional de

Saúde Pública Sergio Arouca,

Fundação Oswaldo Cruz. Rio

de Janeiro RJ Brasil.

${ }^{3}$ Programa de Pós-Graduação

em Enfermagem, Escola de

Enfermagem, Universidade

Federal de Minas Gerais. Belo

Horizonte MG Brasil.

${ }^{4}$ Programa de Pós-Graduação

em Estatística, Departamento

de Estatística, Universidade

Federal de Minas Gerais. Belo

Horizonte MG Brasil.

${ }^{5}$ Programa de Pós-Graduação

em Saúde Pública, Faculdade

de Medicina, Universidade

Federal de Minas Gerais. Belo

Horizonte MG Brasil.

${ }^{6}$ Grupo de Pesquisas em

Epidemiologia e Avaliação em

Saúde, Faculdade de Medicina,

Universidade Federal de Minas

Gerais. Belo Horizonte MG

Brasil.

${ }^{7}$ Instituto René Rachou,

Fundação Oswaldo Cruz. Belo

Horizonte MG Brasil.

${ }^{8}$ Institute for Health Metrics

and Evaluation (IHME),

Universidade de Washington.

Seattle Washington USA.
}

Abstract Mortality indicators for Brazilians aged between 10 and 24 years old were analyzed. Data were obtained from the Global Burden of Disease (GBD) 2019 Study, and absolute numbers, proportion of deaths and specific mortality rates from 1990 to 2019 were analyzed, according to age group (10 to 14, 15 to 19 and 20 to 24 years), sex and causes of death for Brazil, regions and Brazilian states. There was a reduction of $11.8 \%$ in the mortality rates of individuals aged between 10 and 24 years in the investigated period. In 2019, there were 13,459 deaths among women, corresponding to a reduction of $30.8 \%$ in the period. Among men there were 39,362 deaths, a reduction of only $6.2 \%$. There was an increase in mortality rates in the North and Northeast and a reduction in the Southeast and South states. In 2019, the leading cause of death among women was traffic injuries, followed by interpersonal violence, maternal deaths and suicide. For men, interpersonal violence was the leading cause of death, especially in the Northeast, followed by traffic injuries, suicide and drowning. Police executions moved from 77 th to 6 th place. This study revealed inequalities in the mortality of adolescents and young adults according to sex, causes of death, regions and Brazilian states.

Key words Mortality, Adolescent, Young adult, Health status disparities, External causes
Resumo Trata-se de análise de indicadores de mortalidade de brasileiros com idades entre 10 e 24 anos. Foram analisados os dados do Global Burden of Disease (GBD) 2019, utilizando números absolutos, proporção de óbitos e taxas de mortalidade específicas entre 1990 e 2019, segundo faixa etária (10 a 14, 15 a 19 e 20 a 24 anos), sexo e causas de morte para Brasil, regiões e estados brasileiros. Houve redução de 11,8\% nas taxas de mortalidade de indivíduos com idades entre $10 \mathrm{e}$ 24 anos no período investigado. Em 2019, ocorreram 13.459 mortes entre mulheres, correspondendo à redução de 30,8\% no período. Entre homens ocorreram 39.362 óbitos, redução de apenas 6,2\%. Houve aumento das taxas de mortalidade no Norte e Nordeste e redução em estados do Sudeste e Sul. Em 2019, entre mulheres a primeira causa de morte foram lesões por transporte, seguidas por violência interpessoal, mortes maternas e suicídio. Para os homens, a violência interpessoal foi a primeira causa de morte, em especial no Nordeste, seguida das lesões por transporte, do suicídio e dos afogamentos. Execuções policiais passaram do $77^{\circ}$ para o $6^{\circ}$ lugar. Este estudo revelou desigualdades na mortalidade de adolescentes e adultos jovens segundo sexo, causas de óbito, regiões e estados brasileiros.

Palavras-chave Mortalidade, Adolescente, Adulto jovem, Disparidades nos níveis de saúde, Causas externas 


\section{Introdução}

A Organização Mundial de Saúde (OMS) estima que, em 2019, mais de 1,5 milhões de adolescentes e jovens adultos com idades entre 10 e 24 anos morreram no mundo, cerca de 5.000 óbitos por dia ${ }^{1}$. Houve redução das taxas de mortalidade nesse segmento populacional na maioria dos países, à exceção da faixa entre 15 e 19 anos, do sexo masculino, nas regiões do mediterrâneo oriental e das Américas². Países da África apresentam as maiores taxas de anos de vida perdidos ajustados por incapacidade (disability-adjusted life year DALY) e as menores são observadas nos países desenvolvidos ${ }^{2}$.

As principais causas de mortes de indivíduos com idade entre 10 a 24 anos são as agressões, os suicídios e os acidentes de transporte (causas externas), doença mental, uso de álcool, desfechos relacionados à saúde materna e contraceptiva, e doenças infeciosas ${ }^{1-4}$, refletindo insuficiência das políticas públicas de prevenção e proteção ${ }^{3,5}$. A mortalidade entre adolescentes e jovens adultos compromete o futuro e aponta para a negligência de governos e sociedades em garantir a vida plena e com qualidade nesta faixa etária ${ }^{6-9}$.

No Brasil, destaca-se a elevada proporção de mortes por causas externas nesta faixa etária. Em 2016, cerca de metade dos 56 mil óbitos por homicídios ocorreram em jovens com idade entre 15 e 29 anos e, desses, $77 \%$ eram negros ${ }^{10,11}$. Diferenciais no risco de morte de jovens no país também são explicados pelas condições domiciliares e pelas condições de vida nos municípios e nos estados; no Brasil, têm mais risco de morrer aqueles jovens pobres, residentes em áreas mais desenvolvidas ${ }^{12}$.

A "Agenda 2030" para o desenvolvimento sustentável incluiu os adolescentes entre as prioridades, e diversos objetivos incluem ações dirigidas a esse grupo: erradicação da pobreza, igualdade de gênero, acesso à água potável e saneamento, paz e justiça, tornando-se importante monitorar os progressos ${ }^{13,14}$. No Brasil, ainda são escassas as análises de mortalidade de adolescentes e adultos jovens, considerando séries temporais longas. Investigar o padrão de mortalidade nesse grupo etário é ação de grande relevância para a compreensão do fenômeno e o desenvolvimento ou mesmo reorientação de políticas públicas intersetoriais orientadas a esse segmento populacional. Nesse sentido, o presente trabalho visa analisar as taxas de mortalidade em adolescentes e adultos jovens com idades entre 10 e 24 anos, segundo sexo, causas de morte, para Brasil e unidades federadas, entre 1990 e 2019.

\section{Métodos}

Os termos "adolescentes" e "adultos jovens" foram utilizados em referência às pessoas de $10 \mathrm{a}$ 24 anos de idade, faixa etária que inclui o início da adolescência (10 a 14 anos), o meio (15 a 19 anos) e a idade adulta jovem (20 a 24 anos) $)^{15}$.

Foram analisados dados secundários do estudo Global Burden of Disease 2019 (GBD 2019) do Institute for Health Metrics and Evaluation (IHME). Os dados do GBD capturam mortes prematuras e invalidez em decorrência de mais de 350 doenças e lesões em 195 países, por idade e sexo, desde 1990, possibilitando comparar diferentes populações ao longo do tempo ${ }^{16}$. Esses dados são ajustados, o que permite a comparação entre países, bem como com as estimativas subnacionais ${ }^{17,18}$.

O GBD utiliza o Sistema de Informações sobre Mortalidade (SIM), do Ministério da Saúde, como a principal fonte de dados de mortalidade do Brasil, a qual é submetida a ajustes por outras fontes nacionais e internacionais. No caso da mortalidade, são realizados tratamentos estatísticos para melhoria da qualidade das informações. Entre esses tratamentos, são realizadas a redistribuição para códigos garbage - causas que não deveriam ser consideradas como básicas de morte ou causas mal definidas - e a correção para os óbitos não notificados ou com subregistro ${ }^{19}$. O GBD 2019 segue a Classificação Estatística Internacional de Doenças e Problemas Relacionados com a Saúde (CID) 9 e 10 para definição dos grupos de causas. Estes foram descritos previamente $^{17,20}$ e são amplamente revistos a cada nova edição do estudo GBD, em função da inclusão de novas fontes de dados e novas evidências ${ }^{11,19,20}$.

O IHME utiliza os Intervalos de Incerteza de 95\% (II95\%), definidos entre 2,5 e 97,5\% dos valores estimados. Todas as estimativas são calculadas 1000 vezes para obtenção dos II 95\%. Os II $95 \%$ incluem incertezas de todas as fontes e etapas de modelagem, como a variabilidade do tamanho da amostra, dentre outras.

O GBD 2019 organiza a causa básica de morte numa hierarquia de quatro níveis. O nível 1 estratifica as doenças em três grandes grupos: transmissíveis, maternas, neonatais e nutricionais; doenças não transmissíveis; e lesões; os níveis 2, 3 e 4 detalham as doenças do grupo 1, desagregando em 21, 168 e 369 doenças, respectivamente.

Foram analisados o número e a proporção de óbitos, e as taxas de mortalidade para o total da população de adolescentes e adultos jovens com idades entre 10 a 24 anos, segundo Brasil e 
unidade da federação (UF), nos anos de 1990 e 2019, e apresentados os percentuais de mudança dessas estimativas entre os referidos anos. Foram apresentadas as séries históricas do número de óbitos e das taxas de mortalidade entre 1990 a 2019, segundo faixa etária (10 a 14; 15 a 19 e 20 a 24) e sexo, e as variações percentuais das taxas de mortalidade entre os dois anos, também segundo sexo e faixas etárias. Também foram apresentadas as taxas de mortalidade por causa de morte, segundo Brasil, UF e sexo. As causas foram desagregadas no nível 2, 3 ou 4 de hierarquia, segundo sua variação para Brasil, região, UF, faixa etária e ao longo do tempo.

O Estudo Carga Global de Doença no Brasil (GBD Brasil) foi aprovado pelo Comitê de Ética em Pesquisa da Universidade Federal de Minas Gerais.

\section{Resultados}

A Tabela 1 mostra o número de mortes e a taxa de mortalidade por 100 mil habitantes no Brasil e UF. No Brasil, em 1990, no grupo de 10 a 24 anos, houve 51.796 mortes (taxa de 111,1/100 mil; Intervalo de Incerteza - II95\% 109,4 113,/100 mil). Em 2019, foram 49.253 mortes (taxa de 98/100 mil; II95\% 94,8-1001,4/100 mil), redução de $11,8 \%$ no período. No mesmo ano, as taxas mais elevadas ocorreram no Ceará (142,9/100 mil), Pernambuco (139,3/100 mil), Rio Grande do Norte (136,6/100 mil), Espírito Santo (136,1/100 mil) e Sergipe (133,5/100 mil). Maiores reduções entre 1990 e 2019 ocorreram em: São Paulo (-48\%), Rio de Janeiro $(-33,5 \%)$, Distrito Federal (-29\%) e Roraima (-25,8\%). Maiores percentuais de aumento ocorreram no Rio Grande do Norte $(+86,5 \%)$, Ceará $(+74 \%)$, Paraíba $(+50,7 \%)$, Piauí $(+38,5 \%)$, Sergipe $(+37,1 \%)$ e Alagoas $(+24,8 \%)$.

A Figura 1 mostra a variação das taxas de mortalidade entre mulheres de 10 a 24 anos entre 1990 e 2019, por UF (Figura 1A). Em 1990 observou-se taxa de 57,6/100 mil habitantes (13.459 óbitos) e, em 2019, taxa de 39,9/100 mil habitantes (9.891 óbitos)). Houve uma redução das taxas de aproximadamente $30,0 \%$ para o Brasil no período e na maioria dos estados (dados não mostrados).

Conforme a Figura 1, entre os homens ocorreram 38.310 mortes (taxa de 165,1/100 mil) em 1990 (Figura 1B). Em 2019 foram 39.362 (taxa de 154,9/100 mil), com redução de apenas 6,2\% e com grandes disparidades entre as UF. As reduções aconteceram em São Paulo (-54\%), Distrito Federal (-28\%), Rio de Janeiro (-33\%), Rondônia
$(-23 \%)$ e Roraima (-22\%). Em todas as demais UF houve aumento ou estabilidade. Maiores aumentos ocorreram no Rio Grande do Norte $(+122 \%)$, Paraíba $(+70,9 \%)$, Alagoas $(+43 \%)$, Espírito Santo (+38\%), Amapá (+34\%), Pernambuco $(+33 \%)$, Ceará $(+106 \%)$ e Sergipe $(+57 \%)$.

A Figura 2 mostra a série histórica para o número absoluto de mortes (Figura 2A) e taxas de mortalidade por 100.000 habitantes (Figura 2B) nas faixas de 10 a 14, 15 a 19 e 20 a 24 anos, por sexo, no período de 1990 a 2019 . Entre homens de 10 a 14 anos de idade, as mortes passaram de 5.913 (68,0/100 mil; II 95\% 65,8 - 70,4) em 1990 para $3.248(39,2 / 100$ mil; II 95\% 36,1-42,4) em 2019 , com $42,4 \%$ de redução no período. No grupo de 15 a 19 anos, o número de óbitos ficou estável: 13.252 (174,5/100 mil; II 95\% 170,5- 178,8) em 1990 e 14.303 (171,5/100 mil; II 95\% 163,2$179,8)$ em 2019. Na faixa de 20 a 24 anos houve aumento em número absoluto e redução de $10 \%$ na taxa, no período: 19.145 óbitos $(277,1 / 100$ mil; II 95\% 272,3-282,3) em 1990, e 21.811 (248,4/100 mil; II 95\% 240,5 - 257,7) em 2019. Entre mulheres houve redução em todos os grupos de idade. Na faixa de 10 a 14 anos, a redução foi de 37,4\% no período: 3.413 (39,7/100 mil) em 1990 e $1.991(24,8 / 100 \mathrm{mil})$ em 2019 . Entre 15 a 19 anos, foi de 29\%: $4.471(58,1 / 100.000) \mathrm{em}$ 1990 e 3.347 (41,3/100 mil) em 2019. E na faixa de 20 a 24 anos foi de 33,4\%: 5.575 (78,7/100 mil; IC95\%; 77,1 - 80,4) em 1990 e 4.553 mortes (52,4/100 mil; II 95\%: 50,1- 54,9) em 2019.

Conforme mostrado na Figura 3, as séries históricas das regiões indicaram: entre homens, declínio no grupo de 10 a 14 anos e aumento no de 15 a 19 anos até 2017. Redução na faixa de 20 a 24 anos entre 1990 a 2005 e, na sequência, aumento nas regiões Norte, Nordeste, Centro-Oeste, até 2017, seguido de declínio. Na região Sul, houve oscilação em todo o período. No Sudeste, houve redução continua em todo o período. Entre as mulheres, as taxas declinaram nas três faixas etárias em todas as regiões, e aumentaram com a idade.

A Figura 4 mostra as variações percentuais das taxas segundo unidades federadas estratificadas pelas 5 regiões brasileiras, por sexo e faixa etária. A redução média entre homens de 10 a 14 anos foi de $40 \%$, sendo menor no Nordeste (-20\%) e maior no Sudeste (-50\%). Entre 15 a 19 anos houve grande variação regional: aumento próximo a $100 \%$ em estados do Nordeste e redução de $50 \%$ nos estados do Sudeste, o mesmo ocorrendo no grupo de 20 a 24 anos. As mulheres apresentaram menor variabilidade entre faixas etárias e entre estados. Houve redução nos três grupos, em torno de $40 \%$ (10 a 14 e 20 a 24 anos), e cerca de 
Tabela 1. Número de óbitos e taxas de mortalidade em indivíduos com idades entre 10 e 24 anos, ambos os sexos, respectivos Intervalos de Incerteza 95\% e variação percentual dos indicadores, segundo Brasil e unidades federadas, 1990 e 2019.

\begin{tabular}{|c|c|c|c|c|c|c|c|c|c|c|c|c|c|}
\hline \multirow{4}{*}{ Brasil e estados } & \multicolumn{6}{|c|}{ Número de óbitos } & \multicolumn{6}{|c|}{$\begin{array}{c}\text { Taxa de mortalidade } \\
\text { (por } \mathbf{1 0 0 . 0 0 0} \text { habitantes) }\end{array}$} & \multirow{4}{*}{ VP } \\
\hline & \multirow[b]{3}{*}{$\mathbf{n}$} & \multicolumn{2}{|l|}{1990} & \multicolumn{3}{|c|}{2019} & \multicolumn{3}{|c|}{1990} & \multicolumn{3}{|c|}{2019} & \\
\hline & & \multicolumn{2}{|c|}{ II 95\% } & \multirow[b]{2}{*}{$\mathbf{n}$} & \multicolumn{2}{|c|}{ II 95\% } & \multirow[b]{2}{*}{$\mathbf{T x}$} & \multicolumn{2}{|c|}{ II 95\% } & \multirow[b]{2}{*}{$\mathbf{T x}$} & \multicolumn{2}{|c|}{ II 95\% } & \\
\hline & & LI & LS & & LI & LS & & LI & LS & & LI & LS & \\
\hline Brasil & 51769 & 50945 & 52633 & 49253 & 47627 & 50913 & 111,1 & 109,4 & 113,0 & 98,0 & 94,8 & 101,4 & $-11,8$ \\
\hline Acre & 163 & 157 & 169 & 281 & 258 & 304 & 111,9 & 107,8 & 116,1 & 101,2 & 92,9 & 109,5 & $-9,6$ \\
\hline Alagoas & 884 & 841 & 927 & 1219 & 1086 & 1365 & 100,9 & 96,0 & 105,8 & 125,9 & 112,2 & 141,0 & 24,8 \\
\hline Amapá & 100 & 97 & 103 & 305 & 286 & 327 & 103,0 & 99,5 & 106,6 & 118,5 & 110,9 & 126,7 & 15,0 \\
\hline Amazonas & 754 & 708 & 810 & 1183 & 1080 & 1296 & 103,6 & 97,3 & 111,2 & 96,4 & 87,9 & 105,6 & $-7,0$ \\
\hline Bahia & 3747 & 3434 & 4099 & 3190 & 2754 & 3645 & 91,0 & 83,3 & 99,5 & 83,5 & 72,1 & 95,4 & $-8,2$ \\
\hline Ceará & 1720 & 1530 & 1910 & 3445 & 2916 & 4032 & 81,7 & 72,7 & 90,7 & 142,9 & 121,0 & 167,3 & 74,9 \\
\hline Distrito Federal & 649 & 604 & 693 & 605 & 549 & 669 & 120,3 & 111,9 & 128,4 & 85,1 & 77,2 & 94,0 & $-29,3$ \\
\hline Espírito Santo & 920 & 903 & 938 & 1214 & 1084 & 1351 & 110,9 & 108,8 & 113,0 & 136,1 & 121,5 & 151,5 & 22,8 \\
\hline Goiás & 1759 & 1605 & 1926 & 2095 & 1814 & 2424 & 128,3 & 117,1 & 140,5 & 130,1 & 112,7 & 150,5 & 1,4 \\
\hline Maranhão & 1310 & 1161 & 1462 & 1416 & 1196 & 1662 & 78,5 & 69,6 & 87,6 & 63,1 & 53,3 & 74,1 & $-19,6$ \\
\hline Mato Grosso & 832 & 761 & 899 & 931 & 851 & 1016 & 122,4 & 112,1 & 132,3 & 107,9 & 98,6 & 117,8 & $-11,8$ \\
\hline Mato Grosso do Sul & 624 & 597 & 650 & 605 & 553 & 664 & 108,1 & 103,5 & 112,6 & 90,5 & 82,8 & 99,3 & $-16,2$ \\
\hline Minas Gerais & 4518 & 4327 & 4715 & 4348 & 3974 & 4759 & 90,3 & 86,5 & 94,2 & 90,8 & 83,0 & 99,4 & 0,6 \\
\hline Pará & 1648 & 1500 & 1809 & 2488 & 2261 & 2715 & 97,2 & 88,5 & 106,7 & 96,4 & 87,6 & 105,2 & $-0,9$ \\
\hline Paraíba & 830 & 787 & 876 & 1182 & 1050 & 1331 & 76,9 & 72,9 & 81,2 & 115,8 & 102,9 & 130,4 & 50,7 \\
\hline Paraná & 2519 & 2483 & 2557 & 2600 & 2333 & 2886 & 92,5 & 91,2 & 93,9 & 102,7 & 92,2 & 114,0 & 11,1 \\
\hline Pernambuco & 2826 & 2776 & 2881 & 3428 & 3075 & 3774 & 118,0 & 115,9 & 120,3 & 139,3 & 125,0 & 153,4 & 18,1 \\
\hline Piauí & 578 & 549 & 609 & 793 & 721 & 868 & 63,9 & 60,7 & 67,4 & 88,5 & 80,4 & 96,8 & 38,5 \\
\hline Rio de Janeiro & 6585 & 6520 & 6659 & 4567 & 4175 & 4998 & 179,5 & 177,7 & 181,5 & 119,7 & 109,5 & 131,0 & $-33,3$ \\
\hline Rio Grande do Norte & 585 & 537 & 632 & 1184 & 1013 & 1366 & 73,2 & 67,2 & 79,1 & 136,6 & 116,8 & 157,5 & 86,5 \\
\hline Rio Grande do Sul & 2603 & 2569 & 2637 & 2145 & 1959 & 2347 & 101,1 & 99,8 & 102,5 & 90,7 & 82,8 & 99,2 & $-10,3$ \\
\hline Rondônia & 596 & 561 & 633 & 470 & 414 & 533 & 153,0 & 144,1 & 162,6 & 105,5 & 92,9 & 119,6 & $-31,1$ \\
\hline Roraima & 107 & 102 & 112 & 195 & 183 & 208 & 156,1 & 149,3 & 163,2 & 115,9 & 108,5 & 123,6 & $-25,8$ \\
\hline Santa Catarina & 1344 & 1292 & 1401 & 1195 & 1088 & 1316 & 94,6 & 90,9 & 98,7 & 78,0 & 71,1 & 86,0 & $-17,5$ \\
\hline São Paulo & 12824 & 12345 & 13280 & 7012 & 6406 & 7675 & 138,0 & 132,9 & 142,9 & 71,7 & 65,5 & 78,5 & $-48,0$ \\
\hline Sergipe & 500 & 464 & 537 & 810 & 703 & 932 & 97,4 & 90,3 & 104,6 & 133,5 & 115,9 & 153,6 & 37,1 \\
\hline Tocantins & 243 & 222 & 265 & 347 & 306 & 388 & 77,9 & 71,0 & 85,0 & 81,1 & 71,5 & 90,7 & 4,2 \\
\hline
\end{tabular}

20\% (15 a 19 anos). Menores quedas ocorreram no Nordeste e maiores no Sudeste e Sul.

A Figura 5 mostra a mortalidade proporcional por causas de morte, segundo nível 2 do GBD, por sexo, faixa etária e regiões, de 1990 a 2019. Neste período, em todas as regiões brasileiras, entre homens (Figura 5B), principalmente com idades entre 15 e 24 anos, predominam a violência interpessoal, o suicídio e as lesões de trânsito: as violências chegam a cerca de $90 \%$ das causas de óbito. Ao longo do período, reduziram-se as doenças transmissíveis e aumentaram as Doenças Crônicas Não Transmissíveis (DCNT), com diferenças entre faixas etárias e regiões. Em mu- 


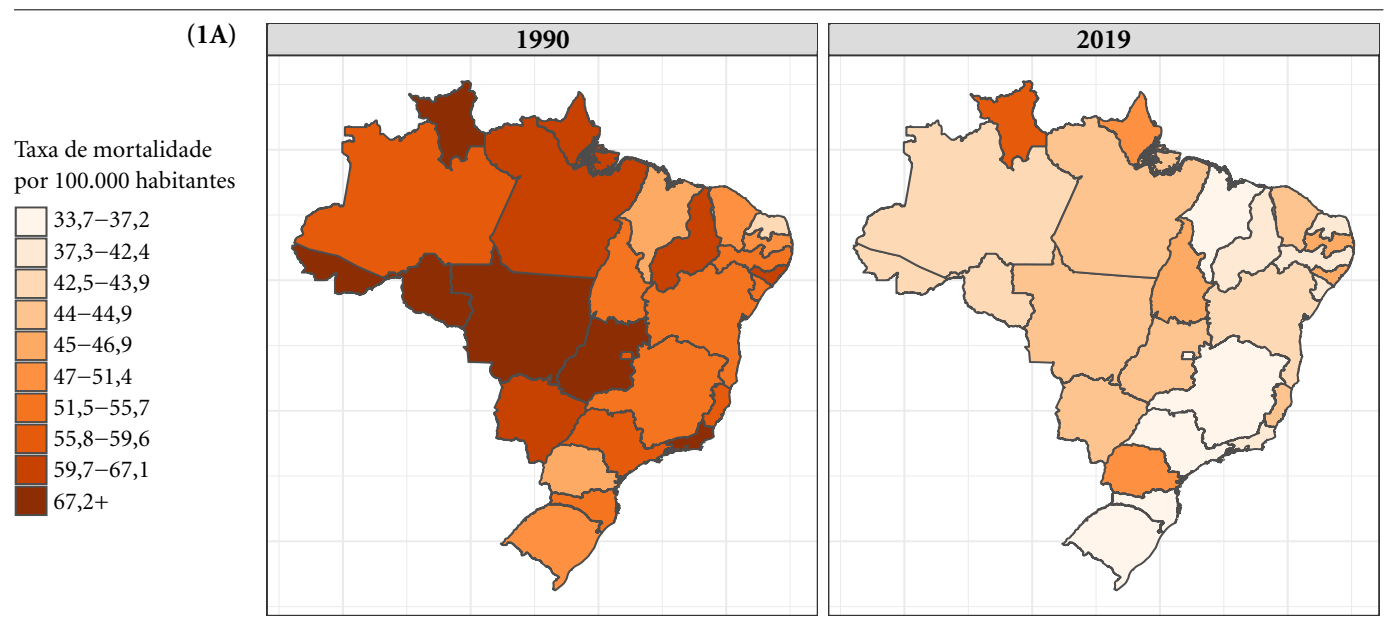

(1B)
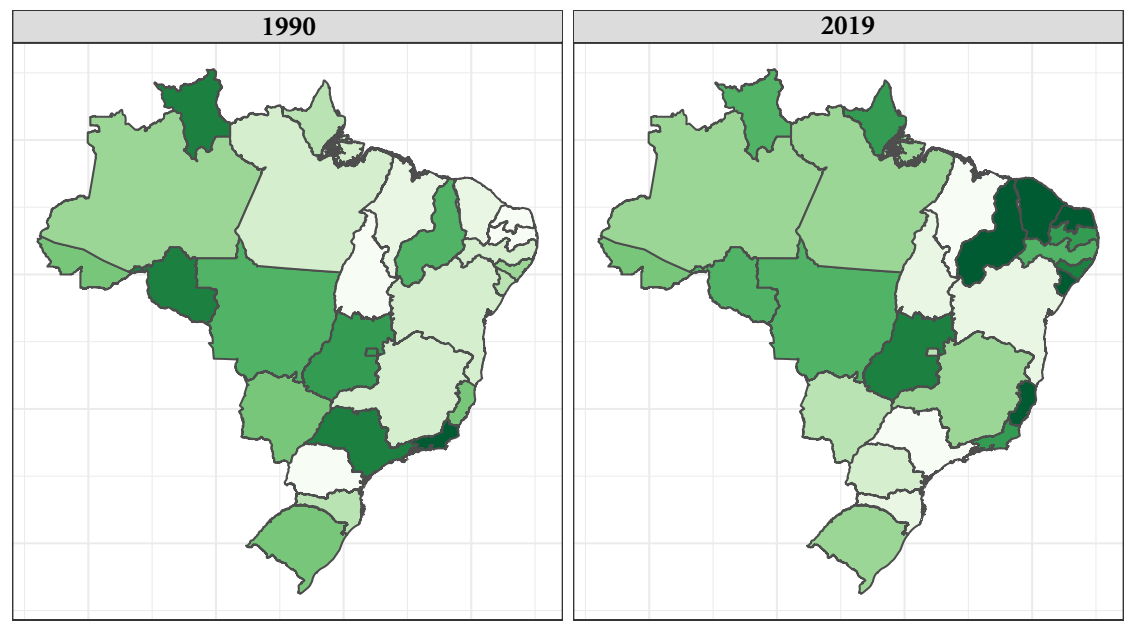

Figura 1. Taxas de mortalidade em indivíduos com idade entre 10 e 24 anos, segundo sexo (1A feminino, 1B masculino), Brasil e unidades federadas, 1990 a 2019.

Fonte: Dados do GBD 2019. Elaboração própria.

lheres (Figura 5A), o perfil de causas assemelhase, mas reduz-se a magnitude das causas externas, que representam cerca de $40 \%$ dos óbitos. As DCNT destacam-se, assim como as mortes maternas e o HIV.

A Figura 6 compara o ranking das 15 primeiras causas de morte para o Brasil em 1990 e 2019, segundo taxas de mortalidade na faixa etária de 10 a 24 anos, por sexo. Em mulheres (Figura 6A), ocorreu redução das taxas de mortalidade no período de 1990 a 2019 até o décimo lugar do ranking, exceto para HIV/Aids. As três primeiras causas em 1990 e 2019 foram: lesões de trânsito, violência interpessoal (agressões) e causas maternas. O suicídio que ocupava o sexto lugar em 1990 passou para o quarto em 2019. As infec- ções respiratórias caíram do quarto (1990) para o quinto (2019); leucemias ocupavam a oitava posição (1990) e passaram para a sexta em 2019; e o sétimo foi ocupado por HIV/Aids. Destacamse oito DCNT nos 15 primeiros lugares em 2019.

Entre os homens (Figura 6B), aumentaram as taxas de violência interpessoal no grupo de 10 a 24 anos, entre 1990 e 2019, de 58/100 mil habitantes para 78,9/100 mil, mantendo-se em primeiro lugar no ranking. A segunda causa de mortes foi por lesões de trânsito, com redução das taxas no período. Os suicídios passaram do quarto para o terceiro lugar, trocando posição com afogamentos que caíram para o $4^{\circ}$ lugar. As infecções respiratórias estão em quinto lugar e as execuções policiais passaram do $77^{\circ}$ lugar 
(2A)

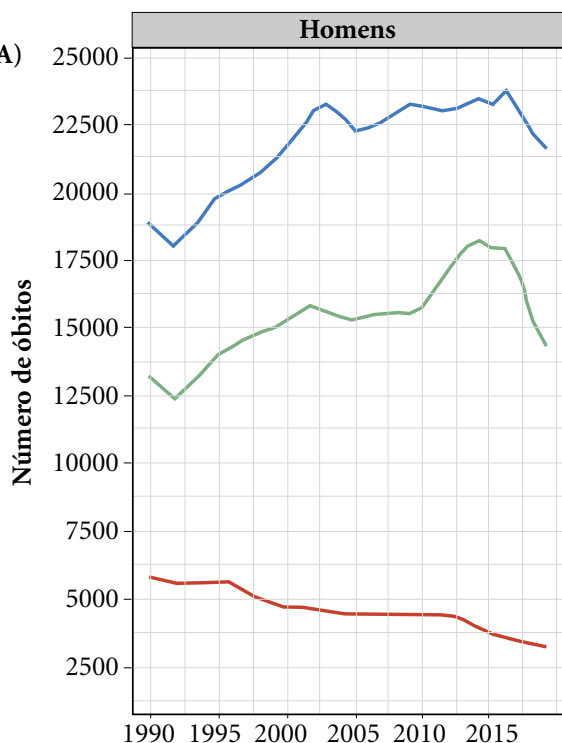

$199019952000 \quad 20052010 \quad 2015$

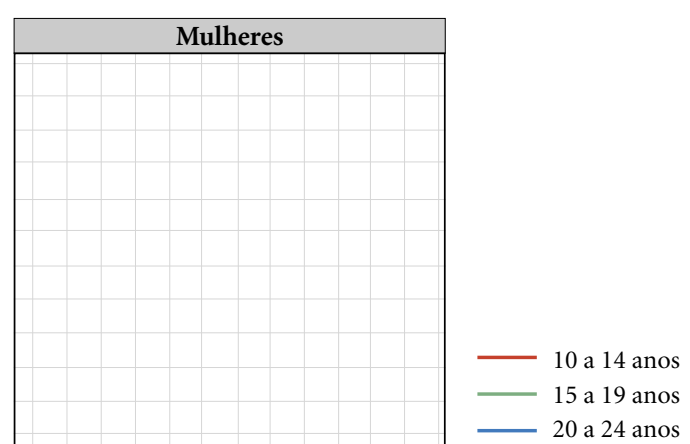

$199019952000 \quad 20052010 \quad 2015$

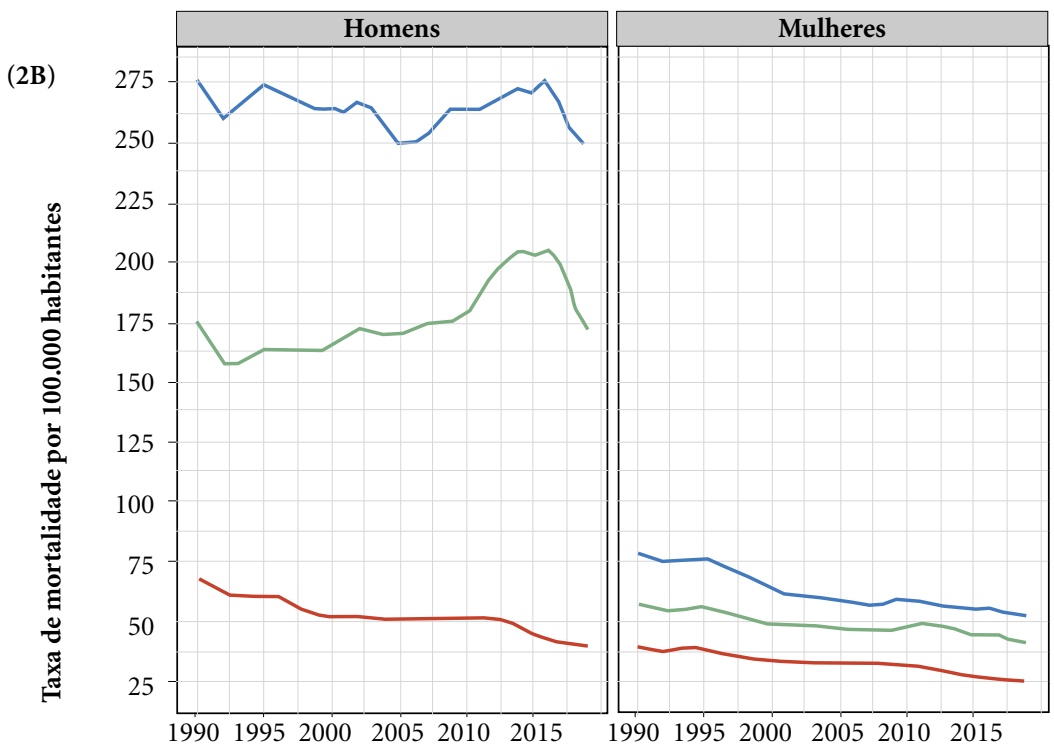

10 a 14 anos
15 a 19 anos

20 a 24 anos

Figura 2. Série histórica do número absoluto (A) e das taxas de mortalidade (B) nas faixas etárias de 10 a 14,15 a 19 e 20 a 24 anos, segundo sexo, Brasil, 1990 a 2019.

Fonte: Dados do GBD 2019. Elaboração própria.

$(0,05 / 100$ mil $)$ para o sexto no período $(2,22 / 100$ mil), o maior salto no ranking das causas de morte entre jovens e adolescentes do sexo masculino.

A Figura 7 mostra as taxas das dez principais causas de morte segundo UF e sexo, em 1990 e 2019. Em mulheres, no ano de 1990 (Figura 7A), as taxas mais elevadas ocorreram por malária nos estados de Rondônia (37/100 mil) e Roraima (33,3/100 mil). As lesões de trânsito foram a primeira ou segunda causa de morte em quase todos os estados. A violência interpessoal ocupa o segundo lugar na maioria dos estados. As causas maternas foram a terceira causa de morte na maioria dos estados, sendo a primeira em Pernambuco 

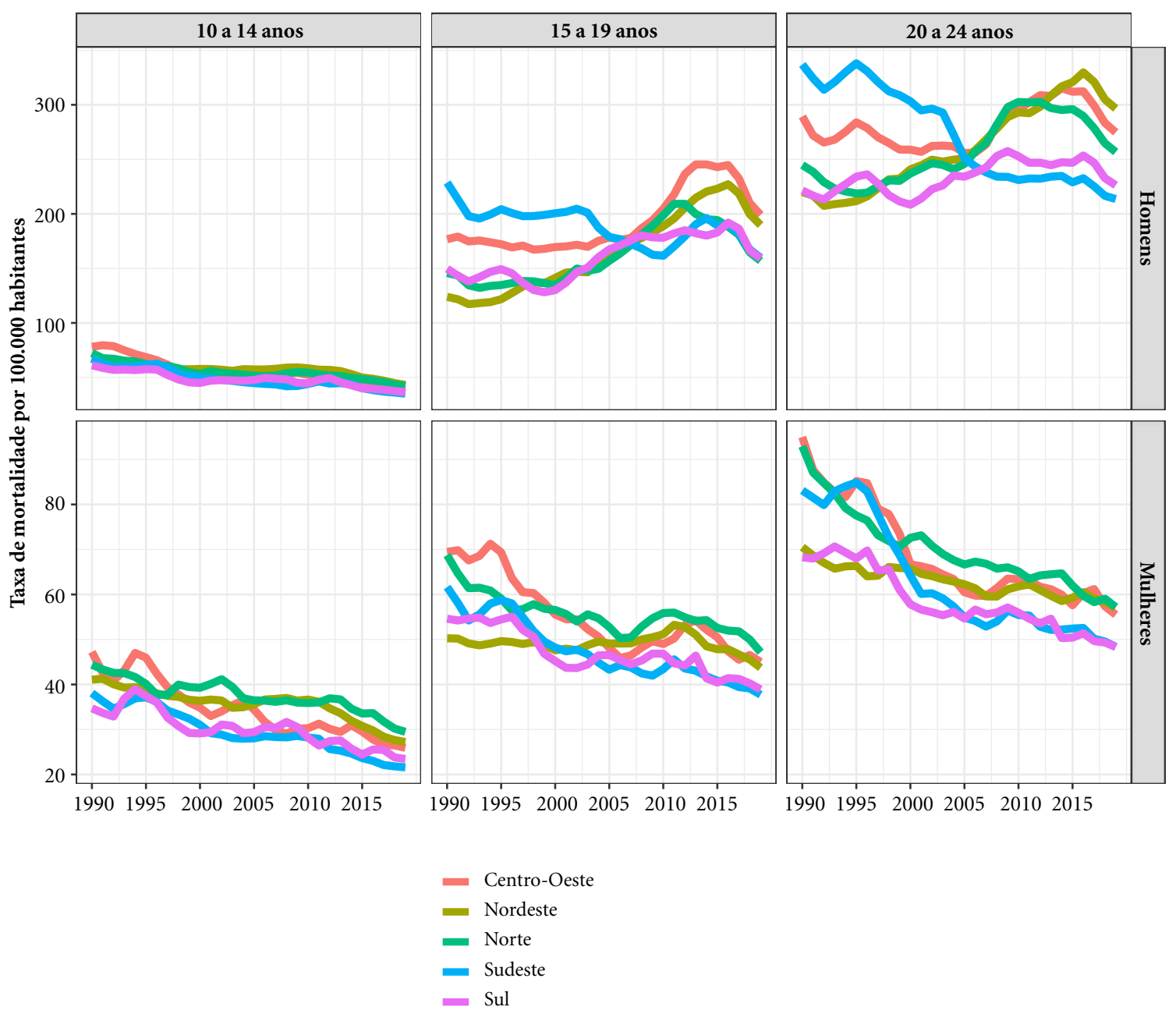

Figura 3. Séries históricas das taxas de mortalidade nas faixas etárias de 10 a 14, 15 a 19 e 20 a 24 anos de idade, segundo sexo e regiões do Brasil, 1990 a 2019.

Fonte: Dados do GBD 2019. Elaboração própria.

(10,1/100 mil). Outras causas foram: infecções respiratórias, afogamentos, suicídios, acidente vascular encefálico, leucemia, afeções congênitas.

Em 2019, houve uma importante mudança do padrão de mortalidade entre mulheres (Figura 7B); a malária, responsável por altas taxas de mortalidade em estados da região Norte e no Mato Grosso em 1990, deixa de constar como uma das dez principais causas de morte. Mantêm-se em primeiro ou segundo lugares as lesões de trânsito, respondendo pelas taxas de mortalidade mais elevadas em Mato Grosso (9,1/100 mil) e Tocantins (9,1/100 mil), e violência interpessoal, que também aparece como primeira ou segunda causa de morte em quase todos os estados, com destaque para Espírito Santo (9,9/100 mil) e Roraima (9,4/100 mil). As causas maternas também se mantiveram como a terceira causa de óbito na maioria dos estados e o suicídio subiu para o quarto lugar na mortalidade. Outras causas foram: infecções respiratórias, leucemias, HIV/Aids, afecções congênitas e acidente vascular encefálico.

Em homens, em 1990 (Figura 7C), em todos os estados, a violência interpessoal liderou as taxas de mortalidade e estas foram maiores no Rio de Janeiro (160,1/100 mil habitantes), em São Paulo (84,5/100 mil) e em Roraima (70,5/100 mil). Em seguida vêm as lesões de trânsito, os afogamentos, os suicídios, outros acidentes, infecções respiratórias, HIV/Aids, quedas, leucemia, acidente vascular cerebral. 


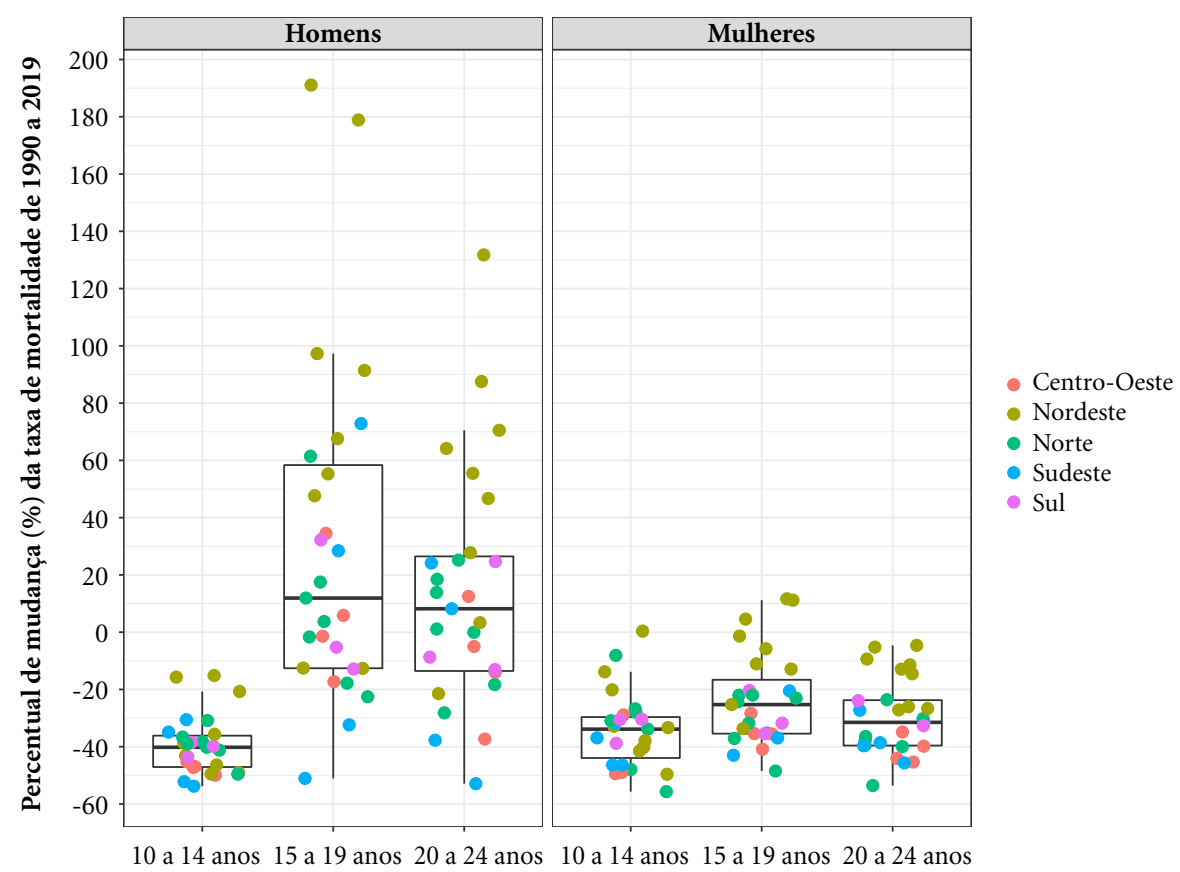

Figura 4. Variação percentual das taxas de mortalidade nas faixas etárias de 10 a 14,15 a 19 e 20 a 24 anos, segundo unidades federadas, estratificadas por região e sexo, 1990 e 2019.

Nota: cada símbolo representa uma unidade federada na respectiva região.

Fonte: Dados do GBD 2019. Elaboração própria.

Também em homens, em 2019 (Figura 7D), em todos os estados, a violência interpessoal continuou a destacar-se como a primeira causa de morte, em especial nos seguintes estados da região Nordeste: Pernambuco $(149,1 / 100.000$ habitantes), Rio Grande do Norte (148,1/100 mil), Ceará (144,6/100 mil), Sergipe (136,3/100 mil), Alagoas (135,7/100 mil), Amapá (106/100 mil), Paraíba (101,6/100 mil), Rio de Janeiro (108,8/100 mil). A menor taxa foi em São Paulo (37,1/100 mil). As lesões de trânsito ficaram em segundo lugar, com taxas variando entre os estados de Mato Grosso (41,7/100 mil habitantes), Rondônia (39,2/100 mil), e Bahia (13/100 mil). Os suicídios subiram para o terceiro lugar no ranking, variando de 11,9/100 mil em Roraima a 3,3/100 mil no Maranhão. Execuções policiais aparecem em sexto lugar. Em seguida vêm os afogamentos, as infecções respiratórias, leucemia, exposição por forças mecânicas, HIV/Aids e outras neoplasias malignas.

\section{Discussão}

O estudo analisou a mortalidade de jovens de $10 \mathrm{a}$ 24 anos nos últimos 30 anos. O número de mortes permaneceu alto em todo o período, cerca de 50 mil adolescentes e jovens morrem anualmente, muitos por causas evitáveis. As taxas de óbito chegam a ser três vezes mais elevadas entre homens em relação a mulheres e apresentam grande variação regional. As causas externas predominam em ambos os sexos, com destaque para violências interpessoais, seguidas por lesões nos transporte, suicídios, e acidentes não intencionais. A violência interpessoal é a principal causa de morte entre os homens jovens. Embora nos últimos 30 anos as taxas de mortalidade por essa causa no sexo masculino tenham reduzido no Sudeste e no Sul do país, observou-se expressivo aumento no Nordeste e no Norte. Destaca-se o crescimento do suicídio em ambos os sexos e, entre homens, as mortes por execuções policiais passaram do $77^{\circ}$ lugar $(0.05 / 100 \mathrm{mil})$ para o sexto $(2,22 / 100 \mathrm{mil})$ no período. Entre as mulheres, as causas maternas encontram-se em terceiro ou quarto lugar, destaca-se o crescimento da mortalidade por neopla- 


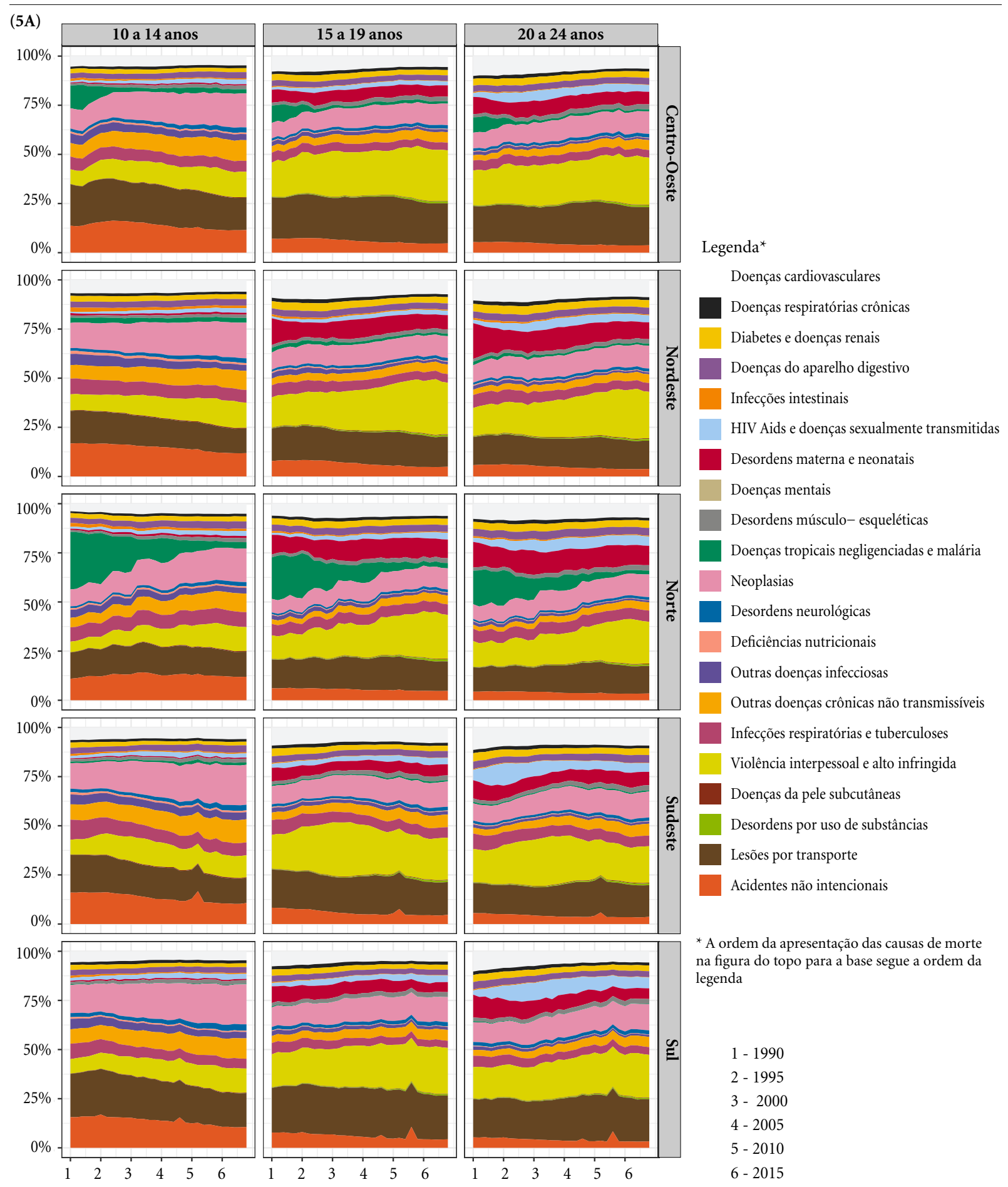

continua

Figura 5. Mortalidade proporcional para principais causas de morte, segundo sexo (5A feminino e 5B masculino), região e faixas etárias de 10 a 14, 15 a 19 e 20 a 24 anos, 1990 a 2019.

sias e doenças cardiovasculares. A mortalidade por doenças como malária e HIV/Aids reduziu.
Segundo o GBD, foram 1,55 milhões de mortes globais de jovens entre 10 e 24 anos em 2017. 


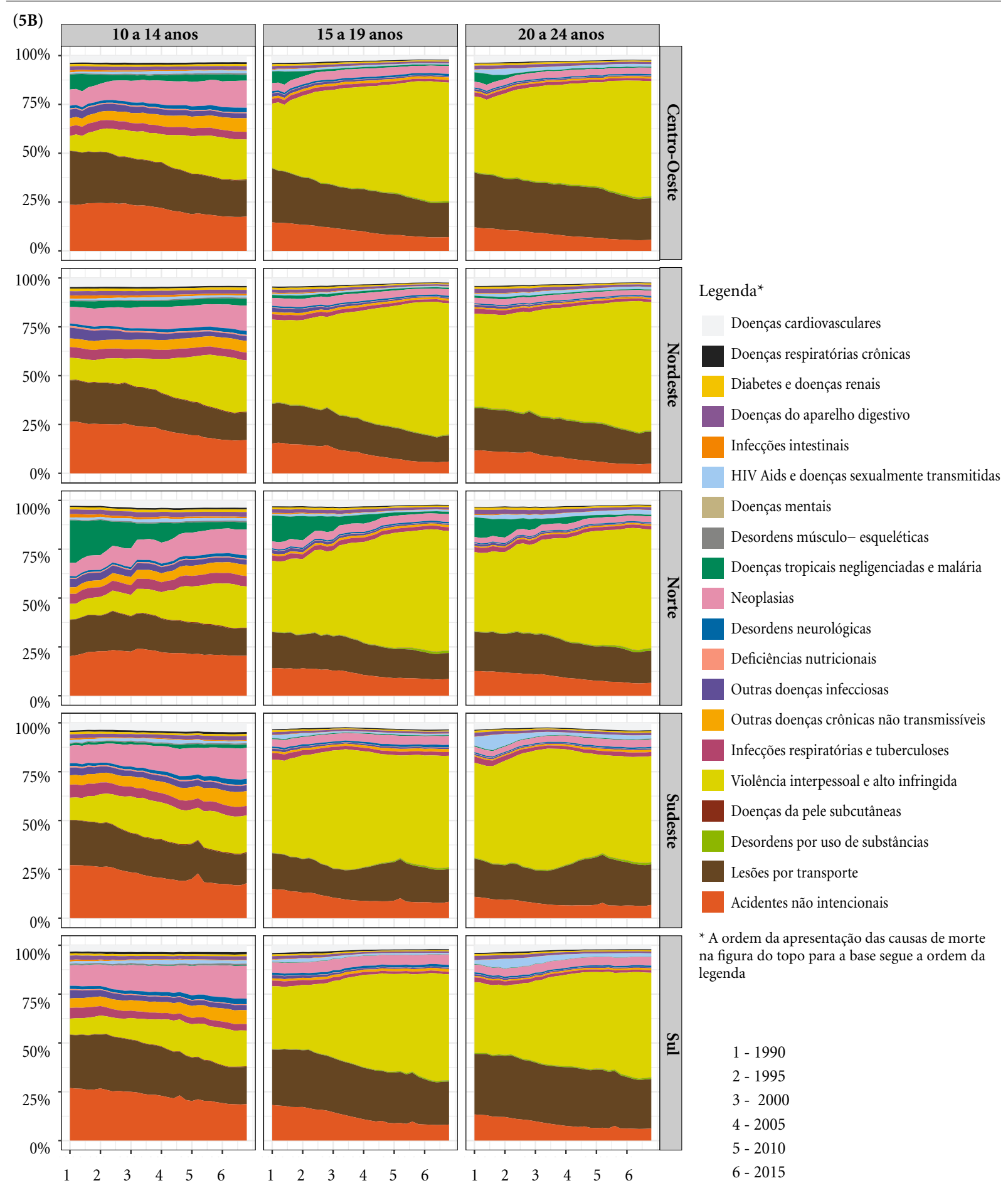

Figura 5. Mortalidade proporcional para principais causas de morte, segundo sexo (5A feminino e 5B masculino), região e faixas etárias de 10 a 14, 15 a 19 e 20 a 24 anos, 1990 a 2019.

Fonte: Dados do GBD 2019. Elaboração própria.

As taxas de mortalidade de adolescentes e adultos jovens apresentam crescimento em diversos países, mostrando uma contínua negligência com esse grupo social ${ }^{3,4,14,21}$. Países como o Brasil e México apresentam redução das taxas de mortalidade na infância, porém têm mantido taxas ele- 
$(6 \mathrm{~A})$

\begin{tabular}{|c|r|l|}
\hline \multicolumn{3}{|c|}{1990} \\
\hline Rank & Taxa & \multicolumn{2}{|c|}{ Causa de Morte } \\
\hline 1 & 9,62 & Acidentes de trânsito \\
\hline 2 & 5,6 & Violência interpessoal \\
\hline 3 & 4,76 & Transtornos maternos \\
\hline 4 & 3,09 & Infeções de vias aéreas inferiores \\
\hline 5 & 2,39 & Afogamento \\
\hline 6 & 2,34 & Autolesão \\
\hline 7 & 2,02 & Doença cerebrovascular \\
\hline 8 & 1,63 & Leucemia \\
\hline 9 & 1,54 & Malária \\
\hline 10 & 1,49 & Anomalias congênitas \\
\hline 11 & 1,23 & Outras neoplasias malignas \\
\hline 12 & 1,19 & Meningite \\
\hline 13 & 1,16 & HIV/AIDS \\
\hline 14 & 1,06 & Doença renal crônica \\
\hline 15 & 1,02 & Doença reumática cardíaca \\
\hline \multicolumn{2}{|l}{} \\
\hline
\end{tabular}

\begin{tabular}{|c|c|c|l|}
\hline & \multicolumn{2}{c|}{2019} \\
\cline { 2 - 5 } & Rank & Taxa & \multicolumn{2}{|c|}{ Causa de Morte } \\
\hline & 1 & 6,18 & Acidentes de trânsito \\
\hline & 2 & 6,03 & Violência interpessoal \\
\hline & 3 & 2,32 & Transtornos maternos \\
\hline & 4 & 2,03 & Autolesão \\
\hline
\end{tabular}

(6B)

\begin{tabular}{|c|c|l|}
\hline 18 & 0,92 & Diabetes mellitus \\
\hline 20 & 0,83 & Outros transtornos musculoesqueléticos \\
\hline 21 & 0,78 & Câncer do cérebro \\
\hline
\end{tabular}

$64 \quad 0,06$ Malária

\begin{tabular}{|c|c|c|c|c|c|}
\hline \multicolumn{3}{|r|}{1990} & \multicolumn{3}{|r|}{2019} \\
\hline Rank & Taxa & Causa de Morte & Rank & Taxa & Causa de Morte \\
\hline 1 & 58,04 & Violência interpessoal & 1 & 78,94 & Violência interpessoal \\
\hline 2 & 34,01 & Acidentes de trânsito & 2 & 26,65 & Acidentes de trânsito \\
\hline 3 & 13,07 & Afogamento & 3 & 6,58 & Autolesão \\
\hline 4 & 6,72 & Autolesão & 4 & 5,38 & Afogamento \\
\hline 5 & 4,53 & Outros ferimentos não intencionais & 5 & 2,43 & Infeções de vias aéreas inferiores \\
\hline 6 & 4,32 & Infeções de vias aéreas inferiores & 6 & 2,22 & Execuções e conflito policial \\
\hline 7 & 3,3 & HIV/AIDS & 7 & 1,88 & Leucemia \\
\hline 8 & 3,28 & Quedas & 8 & 1,83 & Exposição a forças mecânicas \\
\hline 9 & 2,24 & Leucemia & 9 & 1,8 & HIV/AIDS \\
\hline 10 & 2,13 & Doença cerebrovascular & 10 & 1,72 & Outras neoplasias malignas \\
\hline 11 & 2,06 & Exposição a forças mecânicas & 11 & 1,71 & Outros ferimentos não intencionais \\
\hline 12 & 1,95 & Malária & 12 & 1,51 & Quedas \\
\hline 13 & 1,77 & Outros acidentes de transporte & 13 & 1,24 & Câncer do cérebro \\
\hline 14 & 1,74 & Meningite & 14 & 1,22 & Doença isquêmica do coração \\
\hline 15 & 1,73 & $\begin{array}{l}\text { Cirrose e outras doenças hepáticas } \\
\text { crônicas }\end{array}$ & 15 & 1,2 & Anomalias congênitas \\
\hline & & & 16 & 1,19 & Doença cerebrovascular \\
\hline 16 & 1,68 & Outras neoplasias malignas & 17 & 0,97 & Outros acidentes de transporte \\
\hline 17 & 1,5 & Anomalias congênitas & 21 & 0,7 & Cirrose e outras doenças hepáticas \\
\hline 19 & 1,12 & Doença isquêmica do coração & & & crônicas \\
\hline 22 & 0,89 & Câncer do cérebro & 26 & 0,57 & Meningite \\
\hline 77 & 0,05 & Execuções e conflito policial & 73 & 0,06 & Malária \\
\hline
\end{tabular}

Figura 6. Ranking das 15 primeiras causas morte, segundo taxas de mortalidade por 100.000 habitantes na faixa etária de 10 a 24 anos, por sexo (6A feminino, 6B masculino), Brasil, 1990 e 2019.

Fonte: Dados do GBD 2019. Elaboração própria.

vadas de óbitos no grupo etário de adolescentes e adultos jovens ${ }^{22}$.

O Brasil integra grupo dos países com maior risco de morte por homicídio. Os resultados aqui apresentados mostraram taxas elevadas e crescentes, em anos recentes, de homicídios no Norte e Nordeste e importantes reduções no Sudeste, marcadamente em São Paulo e no Rio de Janeiro. 
(7A)

Tocantin
Sergipe
São Paulo
Santa Catarina
Roraima
Rondônia

Rio Grande do Sul

Rio Grande do Norte

Rio de Janeiro Piauí

Pernambuco

Paraná

Paraíba

Pará

Minas Gerais

Mato Grosso do Sul

Mato Grosso

Maranhão

Goiás

Espírito Santo

Distrito Federal

Ceará

Bahia

Amazonas

Amapá

Alagoas

Acre

Brasil

\begin{tabular}{|c|c|c|c|c|c|c|}
\hline 11 & 4,9 & 4,6 & 1,7 & 1,9 & 1,8 & 1,8 \\
\hline 9,4 & 5,3 & 4,3 & 2,5 & 2,6 & 2,4 & 1,5 \\
\hline 11 & 6,2 & 3,6 & 4 & 2,5 & 2,4 & 1,9 \\
\hline 14,1 & 2,8 & 2,3 & 2,3 & 2,5 & 2,9 & 1,8 \\
\hline 9,2 & 6,7 & 2,6 & 2,2 & 2,6 & 2,5 & 1,3 \\
\hline 8,6 & 6,4 & 4,7 & 2,3 & 2,6 & 2,9 & 1,7 \\
\hline 8,3 & 4,7 & 4 & 3 & 2,2 & 3,5 & 1,9 \\
\hline 7,7 & 3,5 & 4,7 & 2,2 & 1,4 & 2,1 & 1,3 \\
\hline 9,9 & 11,3 & 4 & 4,5 & 2 & 2 & 2,8 \\
\hline 7,3 & 2,9 & 5 & 1,9 & 1,9 & 2,1 & 1,6 \\
\hline 8,3 & 7,7 & 10,1 & 2,9 & 2,3 & 2,1 & 2 \\
\hline 11,1 & 3,8 & 5,2 & 2,4 & 2,5 & 3,5 & 1,5 \\
\hline 8,4 & 5,9 & 2,9 & 2,1 & 1,8 & 1,6 & 1,7 \\
\hline 8,2 & 4,9 & 6,5 & 3 & 2 & 1,8 & 2 \\
\hline 8,7 & 3,4 & 4,3 & 3,5 & 3 & 2,6 & 2,5 \\
\hline 11,7 & 6,2 & 5,5 & 3,3 & 2,1 & 4,1 & 2,2 \\
\hline 8,4 & 4,5 & 4,2 & 2 & 2 & 1,9 & 1,6 \\
\hline 7,9 & 4 & 4,1 & 2,2 & 1,4 & 1,5 & 1,6 \\
\hline 15,8 & 8,8 & 4 & 3,5 & 2,7 & 4,9 & 2,4 \\
\hline 11,1 & 7,8 & 3,8 & 2,5 & 3,2 & 1,9 & 2,1 \\
\hline 14,8 & 5 & 3,2 & 2,5 & 1,8 & 2,2 & 1,7 \\
\hline 8,5 & 4,9 & 2,5 & 2,4 & 2,1 & 2,1 & 1,8 \\
\hline 7 & 3,9 & 8 & 2,2 & 2,9 & 1 & 2,3 \\
\hline 6,8 & 4,4 & 5,7 & 2,4 & 2,4 & 1,7 & 1,4 \\
\hline 7,1 & 4,6 & 3,2 & 2,6 & 3,9 & 1,8 & 1,7 \\
\hline 10 & 7,4 & 5,5 & 3 & 2,7 & 1,4 & 2,4 \\
\hline 8,3 & 6,4 & 4,4 & 3,4 & 3,7 & 2,1 & 1,8 \\
\hline 9,6 & 5,6 & 4,8 & 3,1 & 2,4 & 2,3 & 2 \\
\hline 1 & 2 & 3 & 4 & 5 & 6 & 7 \\
\hline
\end{tabular}

\begin{tabular}{|r|r|}
\hline 2,8 & 1,3 \\
\hline 0 & 1,3 \\
\hline 0,1 & 1,7 \\
\hline 0,1 & 1,5 \\
\hline 33,3 & 1,2 \\
\hline 37 & 1 \\
\hline 0,1 & 1,6 \\
\hline 0,1 & 1,2 \\
\hline 0 & 1,6 \\
\hline 0,8 & 1,3 \\
\hline 0 & 1,7 \\
\hline 0,2 & 1,5 \\
\hline 0 & 1,3 \\
\hline 10,8 & 1,2 \\
\hline 0,1 & 1,4 \\
\hline 0,4 & 1,6 \\
\hline 23,5 & 1 \\
\hline 3,1 & 1,2 \\
\hline 0,5 & 2,2 \\
\hline 0,2 & 2 \\
\hline 0,5 & 1,9 \\
\hline 0,3 & 1,3 \\
\hline 0 & 1,2 \\
\hline 10 & 1,2 \\
\hline 18,1 & 1,4 \\
\hline 0 & 1,4 \\
\hline 11,4 & 1,5 \\
\hline 1,5 & 1,5 \\
\hline & \\
\hline
\end{tabular}

$9 \quad 10$
Taxa de mortalidade por 100.000

habitantes, sexo feminino, 1990

$$
0-1,1
$$$$
1,2-1,4
$$

$1,5-1,6$

$1,7-1,9$

$2-2,2$

$2,3-2,7$

$2,8-3,7$

$3,8-4,9$

$5-8,2$

$8,3+$

1 Lesões de trânsito

2 Violência Interpessoal

3 Distúrbios maternos

4 Infecções do trato respiratório interior

5 Afogamento

6 Auto lesão

7 Derrame

8 Leucemia

9 Malária

10 Defeitos congênitos
(7B)

Tocantin
Sergip
São Paulo
Santa Catarin
Roraim
Rondôni

Rio Grande do Sul

Rio Grande do Norte

Rio de Janeiro

Piauí

Pernambuco

Paraná

Paraíba

Pará

Minas Gerais

Mato Grosso do Sul

Mato Grosso

Maranhão

Goiás

Espírito Santo

Distrito Federal

Ceará

Bahia

Amazonas

Amapá

Alagoas

Acre

Brasil
Taxa de mortalidade por 100.000 habitantes, sexo feminino, 2019

$0,6-0,9$

$1-1$

$1,1-1,2$

$1,3-1,3$

$1,4-1,5$

$1,6-1,7$

$1,8-2,4$

$2,5-3,6$

$3,7-6,1$

$6,2+$

1 Lesões de trânsito

2 Violência interpessoal

3 Distúrbios maternos

4 Auto lesão

5 Infecções do trato respiratório interior

6 Leucemia

7 HIV/Aids

8 Outras neoplasias malignas

9 Defeitos congênitos

10 Derrame

Figura 7. Ranking das taxas de mortalidade em indivíduos com idade entre 10 e 24 anos, segundo estado e sexo, em 1990 (7A sexo feminino e 7C sexo masculino) e 2019 (7B sexo feminino e 7D sexo masculino). 
(7C)

Tocantins
Sergipe
São Paulo -
Santa Catarina -
Roraima -
Rondônia -

Rio Grande do Sul

Rio Grande do Norte

Rio de Janeiro

Piauí

Pernambuco

Paraná

Paraíba

Minas

Mato Grosso do Sul

Mato Grosso

Maranhão

Goiás

Espírito Santo

Distrito Federal

Ceará

Bahia

Amazonas

Amapá

Acre

Brasil

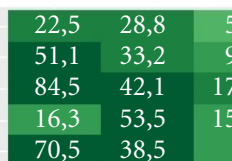

$57,8 \quad 32,1$

$\begin{array}{lll}47,2 & 30,8 & 14,6\end{array}$

$28,7 \quad 26,4$

$160,1 \quad 36,4$

$18,4 \quad 21,9$

$86,4 \quad 26,9$

$28,8 \quad 40,3$

$42,3 \quad 25,2$

$37,9 \quad 24,4$

$21,6 \quad 29,6$

$40,7 \quad 37,6$

$32 \quad 35,6$

$37,9 \quad 27,4$

$48,9 \quad 54,5$

$60,2 \quad 37$

$68,6 \quad 50,3 \quad 10$

\begin{tabular}{l|l}
36,8 & 28,1
\end{tabular}

$35,6 \quad 23,1 \quad 12,4$

$49,4 \quad 24,4 \quad 13,3$

$39,9 \quad 23,4$

58,4

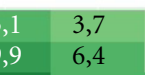

7
7

\begin{tabular}{|l|}
3,3 \\
\hline 2,5 \\
5,4 \\
5,2
\end{tabular}

\begin{tabular}{r|r|r}
5,2 & 3 & 2,3 \\
\hline
\end{tabular}

\begin{tabular}{l|l|}
4,4 & 3,4 \\
5,8 & 2,9
\end{tabular}

$\begin{array}{ll}5,8 & 2,9 \\ 4,8 & 4,4\end{array}$

\begin{tabular}{l|l|l}
3,6 & 2,7 \\
5,1 & 7,5 & 0,6
\end{tabular}

$\begin{array}{ll}3,1 & 7,5\end{array}$

$\begin{array}{lll}2,3 & 1,7 & 0,6\end{array}$

$\begin{array}{rr}4 & 4,4 \\ 2,6 & 3,2\end{array}$

$3,4 \quad 3,6$

$4,3 \quad 4,8$

$\begin{array}{ll}4,8 & 3,9\end{array}$

8

8
3,4

4

4,1

3,7

$\begin{array}{ll}3,6 & 2,5 \\ 6,5 & 2,9\end{array}$

2,9
3,4

2,9
3,4
2,8
3

3

1

\begin{tabular}{r|r|r|r}
\hline 0,7 & 1,4 & 1,4 & 1,2 \\
0,9 & 2,6 & 1,8 & 1,7 \\
\hline 9,7 & 5 & 2,5 & 2,1 \\
\hline 2,3 & 2,2 & 2,5 & 1,7 \\
\hline 2,1 & 2,2 & 1,6 & 1,9 \\
\hline 1,5 & 3 & 1,4 & 1,8 \\
\hline 2,6 & 2,1 & 2,7 & 2,4 \\
\hline 0,6 & 1,4 & 2,1 & 1,2 \\
\hline 4,7 & 5,6 & 2,8 & 3,1 \\
\hline 0,6 & 1 & 1,7 & 1,4 \\
\hline 1,8 & 2,2 & 1,8 & 2,2 \\
1,3 & 2,7 & 2,3 & 1,6 \\
0,3 & 1,3 & 1,9 & 1,4 \\
1,3 & 2,2 & 1,7 & 1,9 \\
1,5 & 3,4 & 2,6 & 2,8 \\
1,8 & 2,3 & 2,1 & 2,1 \\
1,8 & 3,6 & 1,7 & 1,8 \\
0,5 & 0,9 & 1,6 & 1,7 \\
1,5 & 3,1 & 2,6 & 2,3 \\
1,6 & 3,7 & 2,2 & 2,3 \\
\hline 2,1 & 3,8 & 2,6 & 1,9 \\
\hline 0,7 & 1,9 & 2,1 & 1,5 \\
1,1 & 3,5 & 1,7 & 2,1 \\
\hline 1,4 & 2,4 & 1,9 & 1,5 \\
1,7 & 1,6 & 1,7 & 1,5 \\
0,6 & 2 & 1,9 & 1,9 \\
1 & 2,2 & 2,1 & 1,6 \\
\hline 3,3 & 3,3 & 2,2 & 2,1 \\
\hline & & & \\
\hline & 8 &
\end{tabular}

8

9

Taxa de mortalidade por 100.000

habitantes, sexo masculino, 1990

$$
0,3-1,4
$$

$1,5-1,8$

$1,9-2,1$

$2,2-2,7$

$2,8-3,5$

$3,6-4,9$

$5-8,6$

$8,7-16,4$

$16,5-36,3$

$36,4+$

1 Violência interpessoal

2 Lesões de trânsito

3 Afogamento

4 Auto lesão

5 Outras lesões não intencionais

6 Infeccões do trato respiratório interior

7 HIV/AIDS

8 Quedas

9 Leucemia

10 Derrame
(7D)

$$
\begin{array}{r}
\text { Tocantins } \\
\text { Sergipe } \\
\text { São Paulo } \\
\text { Santa Catarina } \\
\text { Roraima } \\
\text { Rondônia }
\end{array}
$$

Rio Grande do Sul

Rio Grande do Norte

Rio de Janeiro

Pernambuco

Paraná

Paraíba

Pará

Minas Gerais

Mato Grosso do Sul

Mato Grosso

Maranhão

Goiás

Espírito Santo

Distrito Federal

Ceará

Amazonas

Amapá

Alagoas

Acre

Brasil

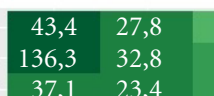

$\begin{array}{lll}37,1 & 23,4 & 6,9\end{array}$

$34,1 \quad 38,9$

$\begin{array}{llr}71,1 & 34,7 & 11,9\end{array}$

$71,2 \quad 39,2$

$69,7 \quad 24,6$

$148,1 \quad 25,3$

$108,8 \quad 24,9$

$41 \quad 37,1$

$149,1 \quad 31,4$

$76,2 \quad 38,5$

$101,6 \quad 34,3$

$84,2 \quad 20,1$

$\begin{array}{llll}87,2 & 28 & 4,5 & 4,9\end{array}$

$\begin{array}{ll}51,9 & 30,2\end{array}$

$73,5 \quad 41,7$

$\begin{array}{rr}40,9 & 18,5 \\ 121,4 & 36,9 \\ 135,9 & 36,5\end{array}$

$\begin{array}{ll}121,4 & 36,9 \\ 135,9 & 36,5\end{array}$

$77,4 \quad 18,4$

$144,6 \quad 36,6$

$\begin{array}{rr}72,6 & 13 \\ 77,4 & 14,4\end{array}$

$106 \quad 18,1$

$\begin{array}{rr}106 & 18,1 \\ 135,7 & 24,2\end{array}$

$77,8 \quad 19,6$

$78,9 \quad 26,6$

1

2

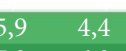

4,4
6,8
5

1,5

$\begin{array}{lll}1,5 & 1,1 & 1,3\end{array}$

\begin{tabular}{l|l|l|}
\hline 2,5 & 0,4 & 2,2 \\
2,9 & 2,8 & 1,8 \\
\hline
\end{tabular}

\begin{tabular}{r|r|r}
1,8 & 1,8 & 2 \\
3,1 & 1 & 1,8
\end{tabular}

$2,7 \quad 0,3$

, 1,7

$\begin{array}{rr}8 & 7,8 \\ 9,2 & 6,1\end{array}$

$8,1 \quad 5,5$

\begin{tabular}{r|r|r}
2,9 & 0,4 & 2,7
\end{tabular}

3,3

$2,3 \quad 0,2 \quad 2$

$2,8 \quad 0,4$

\begin{tabular}{l|l|l}
1,7 & 2,5 & 2,1 \\
2,7 & 0,2 & 2,2
\end{tabular}

$\begin{array}{lll}2,9 & 0,3 & 1,8 \\ 2,2 & 0,4 & 1,8\end{array}$

2,2
2,4

2,4

0,4

$1,6 \quad 1,8$

$0,8 \quad 1,7$

$\begin{array}{ll}0,1 & 1,2 \\ 0,7 & 2,4\end{array}$

$\begin{array}{ll}0,7 & 2,4 \\ 0,5 & 2,1\end{array}$

$\begin{array}{ll}0,3 & 1,7 \\ 0,3 & 2,5\end{array}$

$3 \quad 0,3$

\begin{tabular}{l|l|l|l|l|l}
1,3 & 4,2 & 1,2 & 2,4 & 0,8 & 1,1 \\
2,2 & 0,1 & 1,9 & 1,8 & 3,8 & 1,9
\end{tabular}

\begin{tabular}{|l|l|l|l|l|l|l|}
3,4 & 3,6 & 2,3 & 1,7 & 2 & 2,2 \\
\hline
\end{tabular}

\begin{tabular}{l|l|l|l|l|l|}
\hline 2,6 & 0,2 & 1,6 & 0,9 & 2 & 1,7 \\
\hline
\end{tabular}

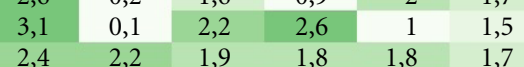

$2,4 \quad 2,2 \quad 1,9$ 
Destaca-se a forte atuação de facções no Norte e Nordeste, que migraram do Sudeste e se instalaram em regiões de fronteiras, associadas ao tráfico de drogas, instalando zonas de conflitos armados, disputas em presídios, promovendo guerras entre facções e acentuando os riscos de mortes nessas regiões ${ }^{23}$. O estudo também aponta para a gravidade das execuções policiais de homens de 10 a 24 anos de idade, comparando-se 1990 e 2019. Dados do Fórum de Segurança Pública apontam que os números oficiais estão subnotifi$\operatorname{cados}^{23} \mathrm{e}$ as execuções ocorrem preferencialmente entre jovens negros, pobres, das periferias urbanas, fruto do racismo estrutural ${ }^{24}$.

As taxas de morte por violência no Brasil estão associadas a desigualdades estruturais, ausência de políticas sociais eficazes e efetivas para os jovens, desemprego, conflitos em áreas de fronteiras agrícolas, disputas por terra e políticas de segurança pública inadequadas ${ }^{25,26}$. Os homicídios de adolescentes e adultos jovens têm forte associação com o abandono escolar, antecedentes criminais, tráfico e uso de drogas, abuso de álcool, abusos e violência doméstica ${ }^{27}$.

As desigualdades de gênero são destacadas no caso da violência. Homens jovens têm taxas de mortalidade até quatro vezes mais elevadas do que as mulheres jovens, o que também se observa em estudos globais ${ }^{4}$. As diferenças nas mortes violentas segundo sexo, citadas na literatura, ressaltam aspectos culturais que incentivam comportamentos e atitudes agressivas dos homens desde a infância, envolvimento com drogas, armas, crime organizado e sua maior exposição a práticas de risco ${ }^{26}$. Entretanto, as taxas de violências contra meninas e mulheres no Brasil são mais elevadas que na maioria dos países do mundo. Relatório da ONU aponta que o Brasil ocupa o quinto lugar global em feminicídios em decorrência da violência sexual e do machismo ${ }^{27}$. Estudos destacam que as mulheres assassinadas são em geral jovens, negras, solteiras, de baixa escolaridade e renda ${ }^{28}$, embora o feminicídio ocorra em todas as classes sociais.

As lesões de trânsito resultam em aproximadamente 1,35 milhão de mortes em todo o mundo e representam a principal causa de morte de crianças e jovens de 5 a 29 anos de idade no planeta ${ }^{29}$. $\mathrm{O}$ presente estudo aponta taxas de mortalidade por lesões de trânsito elevadas em ambos os sexos e, na maioria dos estados, essas representam a segunda ou a terceira causa de morte, o que sugere necessidade de ações de prevenção e de promoção da saúde relacionadas ao trânsito. No Brasil, o rápido crescimento econômico e o acesso a compra de motocicletas produziu uma grande elevação das taxas de mortalidade na faixa etária estudada. $\mathrm{O}$ maior risco se alia ao não uso de capacete, baixa fiscalização, falhas na segurança e problema de infraestrutura de transporte ${ }^{30}$. As diferencas entre estados na mortalidade por acidente de trânsito são proeminentes, sendo mais elevadas no Centro-Oeste e no Nordeste, sugerindo menor capacidade de intervenção e regulação do estado nessas regiões ${ }^{31}$ e baixo investimento em ações de prevenção ${ }^{32}$.

$\mathrm{O}$ estudo indica aumento da importância do suicídio entre jovens, que subiu posições no ranking e se posiciona como terceira causa de morte de homens e quarta causa de mulheres. Dados globais mostram que as lesões autoinflingidas são crescentes e uma das principais causas de mortalidade de adolescentes na Europa e Ásia ${ }^{4}$. Diversos fatores podem explicar este aumento como: transtornos mentais, abuso de substâncias, falta de perspectiva com o futuro, desemprego, violências, isolamento social ou geográfico, baixo acesso a cuidados clínicos ${ }^{33}$, abusos físicos e sexuais e dificuldades com a orientação sexual ${ }^{34,35}$. Dentre as medidas apontadas para sua redução, destaca-se a importância da ampliação do acesso aos equipamentos de saúde mental, suporte familiar, redução das desigualdades, além de restrição do acesso a armas de fogo e produtos químicos letais ${ }^{10,36}$.

As causas maternas são importantes causas de morte global e, somadas às causas de morte transmissíveis, chegam a um terço das mortes entre adolescentes e adultos jovens com idades entre 10 e 24 anos $^{4}$. O estudo aponta que, embora tenham caído as taxas, as causas maternas se mantêm em terceiro lugar referente às mortes de mulheres, o que reflete falta de acesso a métodos contraceptivos e a serviços de saúde ${ }^{37}$. As diferenças regionais com relação às mortes maternas são expressivas; as taxas mais elevadas estão no Nordeste. Superar as desigualdades no acesso aos serviços de saúde e as condições sociais em que as mulheres jovens vivem é fundamental para melhorar a saúde das adolescentes e mães.

Outra importante causa de morte é o HIV/ Aids, para a qual se constatou aumento das taxas entre as mulheres. Atualmente ocupa o sétimo lugar das causas de óbito entre as mulheres e a nona posição entre os homens. Em 2019, estados como Roraima e Rio de Janeiro apresentam taxas elevadas entre as mulheres (quarto lugar), o que deve ser investigado tanto em relação à transmissão vertical mãe/filho ocorrida no passado, quanto em relação à prostituição e ao uso de drogas entre adolescentes e adultos joven ${ }^{38}$. Análises de dados do SINAN apontam para a redução da mortalidade e incidência por esta causa na população geral, exceto no grupo de 10 a 29 
$\operatorname{anos}^{38}$. A maior incidência nas referidas faixas de idade sugere falha nas medidas de prevenção, o que pode comprometer toda uma geração. Dentre as omissões e falhas, destaca-se a redução do investimento em comunicação, ações de prevenção, distribuição de preservativos, entre outras ${ }^{39}$. Dados da PENSE, apontam redução de uso de preservativos no período de 2009 a 2015, entre adolescentes de 13 a 15 anos, bem como redução de comunicação nas escolas sobre saúde sexual e acesso a preservativos ${ }^{37,39}$, o que também sugere déficit na condução de ações programáticas de educação em saúde e promoção da saúde, e aumento de posturas conservadoras e e de cunho religioso na sociedade ${ }^{39}$.

Destacam-se os progressos em relação às doenças tropicais, como a malária no Norte e outras doenças infeciosas negligenciadas no Nordeste e Centro-Oeste. Esforço e vitória do Sistema Único de Saúde e da ciência em prover vacinas, tratamento, ações de vigilância e de controle ${ }^{40}$.

Os dados apontam ainda para o crescimento das DCNT, como doenças cardiovasculares, câncer, incluindo leucemia, e outras doenças que refletem efeitos das mudanças nos estilos de vida, consumo de alimentos ultraprocessados, inatividade fisica, poluição, irradiação ${ }^{41}$, mas também efeito de herança genética e envelhecimento da população.

Os dados do estudo GBD têm limitações inerentes à produção de estimativas de mortalidade e relacionadas às fontes utilizadas. No caso do Brasil, a fonte de dados do GBD é o SIM que, embora tenha ampliado a captação de registros e melhorado sua qualidade em anos recentes, no passado e ainda em alguns estados possui óbitos não captados, registros incompletos e elevada proporção de códigos garbage. A análise da carga global de mortalidade de adolescentes utiliza dados modelados e as estimativas que relatamos aqui precisam ser vistas dentro desse contexto.

A redução da mortalidade de adolescentes requer enfrentar as desiguadades sociais, econômicas, culturais e investir na educação, emprego e inclusão social ${ }^{7,12,42}$. Embora o Estatuto da Criança e do Adolescente (ECA) reconheça os adolescentes como sujeitos de direitos, passados 30 anos do ECA, o país ainda não consegue proteger esta população. Os adolescentes e adultos jovens não desfrutam da redução acelerada da mortalidade observada em crianças mais jovens ${ }^{8}$.

A Agenda 2030 representa uma chamada ao país para priorizar políticas públicas que ampliem os direitos de crianças e adolescentes e garantam condições de desenvolvimento infanto-juvenil ${ }^{23}$, investindo em equidade e na eliminação da pobreza extrema, da fome e do racismo; ofertando saúde e educação de qualidade; e promovendo sociedades pacíficas e inclusivas. São compromissos com o presente e o futuro, visando à redução de diferenças entre ricos e pobres e da carga morbimortalidade nessa faixa etária, em especial por causas externas ${ }^{43}$.

\section{Considerações finais}

Este estudo revelou profundas desigualdades na mortalidade de adolescentes e adultos jovens segundo sexo, causas, regiões e unidades federadas brasileiras. Os progressos para melhorar a saúde dos jovens têm sido lentos e ainda há grandes desafios para enfrentar a violência, os ambientes inseguros, a mortalidade materna e as falhas nos programas de contracepção. Apesar do crescente reconhecimento da importância da saúde do adolescente e dos adultos jovens para o desenvolvimento e o crescimento econômico futuro, ao apresentar as causas de mortalidade o estudo aponta também para os riscos à saúde neste ciclo de vida. Há necessidade de se desenvolverem abordagens para lidar com as crescentes desigualdades na mortalidade nessa faixa etária.

$\mathrm{Na}$ arena política, vêm ganhando força pautas como a redução da maioridade penal e a flexibilização do Estatuto do Desarmamento. Considerase que tais medidas, se aprovadas, podem afetar ainda mais o futuro da juventude brasileira, que precisa, ao contrário, de medidas protetivas e que fomentem a cultura da paz e a não violência. Inclusão social, educação de qualidade e acesso a emprego são a direção apontada por aqueles que trabalham com esse grupo etário e se preocupam com o futuro do país.

\section{Colaboradores}

DC Malta e MCS Minayo conceberam o estudo e elaboraram a versão preliminar. GA Veloso realizou a extração dos dados. Todos os autores realizaram a interpretação dos dados, revisaram criticamente, contribuíram para a versão final do manuscrito e a aprovaram.

\section{Financiamento}

Secretaria de Vigilância em Saúde, Ministério da Saúde, TED 148-2018, projeto "Desigualdades em pequenas áreas geográficas dos indicadores de doenças crônicas não transmissíveis, violências e seus fatores de risco". 


\section{Referências}

1. World Health Organization (WHO) [Internet]. Geneva: WHO; 2021 [cited 2021 Jan 28]. Adolescent and young adult health. Available from: https://www.who. int/news-room/fact-sheets/detail/adolescents-health -risks-and-solutions.

2. World Health Organization (WHO) (b) [Internet]. Geneva: WHO; c2021 [cited 2021 Jan 28]. Adolescent health epidemiology. Available from: https://www. who.int/maternal_child_adolescent/epidemiology/ adolescence/en/.

3. Mokdad AH, Forouzanfar MH, Daoud F, Mokdad AA, El Bcheraoui C, Moradi-Lakeh M, Kyu HH, Barber RM, Wagner J, Cercy K, Kravitz H, Coggeshall M, Chew A, O’Rourke KF, Steiner C, Tuffaha M, Charara R, Al-Ghamdi EA, Adi Y, Afifi RA, Alahmadi H, AlBuhairan F, Allen N, AlMazroa M, Al-Nehmi AA, AlRayess Z, Arora M, Azzopardi P, Barroso C, Basulaiman M, Bhutta ZA, Bonell C, Breinbauer C, Degenhardt L, Denno D, Fang J, Fatusi A, Feigl AB, Kakuma R, Karam N, Kennedy E, Khoja TA, Maalouf F, Obermeyer CM, Mattoo A, McGovern T, Memish ZA, Mensah GA, Patel V, Petroni S, Reavley N, Zertuche DR, Saeedi M, Santelli J, Sawyer SM, Ssewamala F, Taiwo K, Tantawy M, Viner RM, Waldfogel J, Zuñiga MP, Naghavi M, Wang H, Vos T, Lopez AD, Al Rabeeah AA, Patton GC, Murray CJ. Global burden of diseases, injuries, and risk factors for young people's health during 1990-2013: a systematic analysis for the Global Burden of Disease Study 2013. Lancet [serial on the Internet]. 2016 [cited $2020 \mathrm{Nov}$ 26];387(10036):[about 18 p.]. Available from: https:// www.thelancet.com/journals/lancet/article/PIIS01406736(16)00648-6/fulltext.

4. GBD 2017 Child and Adolescent Health Collaborators. Diseases, injuries, and risk factors in child and adolescent health, 1990 to 2017: findings from the Global Burden of Diseases, Injuries, and Risk Factors 2017 Study. JAMA pediatr [serial on the Internet]. 2019 [cited 2020 Nov 26];173(6):e190337. Available from: https://jamanetwork.com/journals/jamapediatrics/fullarticle/2732143.

5. Malta DC, Saltarelli RMF, Prado RR, Monteiro RA, Almeida MF. Mortes evitáveis no Sistema Único de Saúde na população brasileira, entre 5 e 69 anos, 2000-2013. Rev Bras Epidemiol 2018; 21:e180008.

6. Patton GC, Coffey C, Sawyer SM, Viner RM, Haller DM, Bose K, Vos T, Ferguson J, Mathers CD. Global patterns of mortality in young people: a systematic analysis of population health data. Lancet 2009 [cited 2020 Nov 26]; 374(9693):[about 11 p.]. Available from: https://www.thelancet.com/journals/lancet/article/PIIS0140-6736(09)60741-8/fulltext.

7. Azzopardi PS, Hearps SJC, Francis KL, Kennedy EC, Mokdad AH, Kassebaum NJ, Lim S, Irvine CMS, Vos T, Brown AD, Dogra S, Kinner SA, Kaoma NS, Naguib M, Reavley NJ, Requejo J, Santelli JS, Sawyer SM, Skirbekk V, Temmerman M, Tewhaiti-Smith J, Ward JL, Viner RM, Patton GC. Progress in adolescent health and wellbeing: tracking 12 headline indicators for 195 countries and territories, 1990-2016. Lancet [serial on the Internet]. 2019 [cited 2020 Nov 26]; 393(10176):[about 17 p.]. Available from: https:// www.thelancet.com/journals/lancet/article/PIIS01406736(18)32427-9/fulltext.
8. The Lancet Child \& Adolescent Health. Universal health coverage and the forgotten generation. Lancet Child Adolesc Health [serial on the Internet]. 2019 Nov [cited 2020 Nov 30];3(11):[about 1 p.]. Available from: https://www.thelancet.com/journals/lanchi/ article/PIIS2352-4642(19)30299-8/fulltext.

9. GBD 2019 Diseases and Injuries Collaborators. Global burden of 369 diseases and injuries in 204 countries and territories, 1990-2019: a systematic analysis for the Global Burden of Disease Study 2019. Global Health Metrics. Lancet 2020; 396(10258):1204-1222.

10. Malta DC, Soares Filho AM, Pinto IV, de Souza Minayo MC, Lima CM, Machado ÍE, Teixeira RA, Neto OLM, Ladeira RM, Merchan-Hamann E, de Souza MFM, Vasconcelos CH, Vidotti CCF, Cousin E, Glenn S, Bisignano C, Chew A, Ribeiro AL, Naghavi M. Association between firearms and mortality in Brazil, 1990 to 2017: a global burden of disease Brazil study. Popul Health Metr [serial on the Internet] 2020 Set [cited 2020 Dez 9]; 18(Supl. 1):19. Available from: https://www.springermedizin.de/association-between-firearms-and-mortality-in-brazil1990-to-201/18433952?fulltextView=true.

11. Brasil. Ministério da Saúde (MS). Secretaria de Vigilância em Saúde. Departamento de Vigilância de Doenças e Agravos não Transmissível e Promoção da Saúde. Saúde Brasil 2017: uma análise da situação de saúde e os desafios para o alcance dos objetivos de desenvolvimento sustentável. Brasília: MS; 2018.

12. Pereira FNA, Queiroz BL. Diferenciais de mortalidade jovem no Brasil: a importância dos fatores socioeconômicos dos domicílios e das condições de vida nos municípios e estados brasileiros. Cad Saúde Publica 2016; 32(9):e00109315.

13. GBD 2017 Mortality Collaborators. Global, regional, and national age-sex-specific mortality and life expectancy, 1950-2017: a systematic analysis for the Global Burden of Disease Study 2017. Lancet [serial on the Internet]. 2018 Nov [cited 2020 Nov 30]; 392(10159):[about 51 p.]. Available from: https:// www.thelancet.com/journals/lancet/article/PIIS01406736(18)31891-9/fulltext.

14. Masquelier B, Hug L, Sharrow D, You D, Hogan D, Hill K, Liu J, Pedersen J, Alkema L, United Nations Inter-agency Group for Child Mortality Estimation. Global, regional, and national mortality trends in older children and young adolescents (5-14 years) from 1990 to 2016: an analysis of empirical data. Lancet Glob Health [serial on the Internet]. 2018 Out [cited 2020 Nov 30]; 6(10):[about 12 p.]. Available from: https://www.thelancet.com/journals/langlo/article/ PIIS2214-109X(18)30353-X/fulltext.

15. Brasil. Ministério da Saúde (MS). Saúde e desenvolvimento da juventude brasileira: construindo uma agenda nacional. Brasília: MS; 1999. [acessado 2021 abr. 3]. Disponível em: http://bvsms.saude.gov.br/bvs/publicacoes/saude_juventude.pdf.

16. Institute for Health Metrics and Evaluation (IHME) [Internet]. Seattle: IHME; c2020 [cited 2021 Jan 29]. Data Visualization. Available from: http://www.healthdata.org/results/data-visualizations. 
17. Lozano R, Naghavi M, Foreman K, Lim S, Shibuya K, Aboyans V, Abraham J, Adair T, Aggarwal R, Ahn SY, Alvarado M, Anderson HR, Anderson LM, Andrews KG, Atkinson C, Baddour LM, Barker-Collo S, Bartels DH, Bell ML, Benjamin EJ, Bennett D, Bhalla K, Bikbov B, Bin Abdulhak A, Birbeck G, Blyth F, Bolliger I, Boufous S, Bucello C, Burch M, Burney P, Carapetis J, Chen H, Chou D, Chugh SS, Coffeng LE, Colan SD, Colquhoun S, Colson KE, Condon J, Connor MD, Cooper LT, Corriere M, Cortinovis M, de Vaccaro KC, Couser W, Cowie BC, Criqui MH, Cross M, Dabhadkar KC, Dahodwala N, De Leo D, Degenhardt L, Delossantos A, Denenberg J, Des Jarlais DC, Dharmaratne SD, Dorsey ER, Driscoll T, Duber H, Ebel B, Erwin PJ, Espindola P, Ezzati M, Feigin V, Flaxman AD, Forouzanfar MH, Fowkes FG, Franklin R, Fransen M, Freeman MK, Gabriel SE, Gakidou E, Gaspari F, Gillum RF, Gonzalez-Medina D, Halasa YA, Haring D, Harrison JE, Havmoeller R, Hay RJ, Hoen B, Hotez PJ, Hoy D, Jacobsen KH, James SL, Jasrasaria R, Jayaraman S, Johns N, Karthikeyan G, Kassebaum N, Keren A, Khoo JP, Knowlton LM, Kobusingye O, Koranteng A, Krishnamurthi R, Lipnick M, Lipshultz SE, Ohno SL, Mabweijano J, MacIntyre MF, Mallinger L, March L, Marks GB, Marks R, Matsumori A, Matzopoulos R, Mayosi BM, McAnulty JH, McDermott MM, McGrath J, Mensah GA, Merriman TR, Michaud C, Miller M, Miller TR, Mock C, Mocumbi AO, Mokdad AA, Moran A, Mulholland K, Nair MN, Naldi L, Narayan KM, Nasseri K, Norman P, O’Donnell M, Omer SB, Ortblad K, Osborne R, Ozgediz D, Pahari B, Pandian JD, Rivero AP, Padilla RP, Perez-Ruiz F, Perico N, Phillips D, Pierce K, Pope CA 3rd, Porrini E, Pourmalek F, Raju M, Ranganathan D, Rehm JT, Rein DB, Remuzzi G, Rivara FP, Roberts T, De León FR, Rosenfeld LC, Rushton L, Sacco RL, Salomon JA, Sampson U, Sanman E, Schwebel DC, Segui-Gomez M, Shepard DS, Singh D, Singleton J, Sliwa K, Smith E, Steer A, Taylor JA, Thomas B, Tleyjeh IM, Towbin JA, Truelsen T, Undurraga EA, Venketasubramanian N, Vijayakumar L, Vos T, Wagner GR, Wang M, Wang W, Watt K, Weinstock MA, Weintraub R, Wilkinson JD, Woolf AD, Wulf S, Yeh PH, Yip P, Zabetian A, Zheng ZJ, Lopez AD, Murray CJ, AlMazroa MA, Memish ZA. Global and regional mortality from 235 causes of death for 20 age groups in 1990 and 2010: a systematic analysis for the Global Burden of Disease Study 2010. Lancet [serial on the Internet] 2012 Dez [cited 2020 Nov 30]; 380(9859):[about 33 p.]. Available from: https://www.thelancet.com/article/S01406736(12)61728-0/fulltext\#.

18. GBD 2013 Mortality and Causes of Death. Global, regional, and national age-sex specific all-cause and cause-specific mortality for 240 causes of death, 19902013: a systematic analysis for the Global Burden of Disease Study 2013. Lancet [serial on the Internet] 2015 Jan [cited 2020 Nov 30]; 385(9963):[about 68 p.]. Available from: https://www.thelancet.com/journals/lancet/article/PIIS0140-6736(14)61682-2/fulltext.
19. GBD 2017 Causes of Death Collaborators. Global, regional, and national age-sex-specific mortality for 282 causes of death in 195 countries and territories, 19802017: a systematic analysis for the Global Burden of Disease Study 2017. Lancet 2018; 392(10159):17361788.

20. GBD 2019 Demographics Collaborators. Global agesex-specific fertility, mortality, healthy life expectancy (HALE), and population estimates in 204 countries and territories, 1950-2019: a comprehensive demographic analysis for the Global Burden of Disease Study 2019. Lancet 2020; 396(10258):1160-1203.

21. Viner RM, Coffey C, Mathers C, Bloem P, Costello A, Santelli J, Patton GC. 50-year mortality trends in children and young people: a study of 50 low-income, middle-income, and high-income countries. Lancet 2011; 377(9772):1162-1174.

22. GBD 2019 Diseases and Injuries Collaborators. Global burden of 369 diseases and injuries in 204 countries and territories, 1990-2019: a systematic analysis for the Global Burden of Disease Study 2019. Lancet 2020; 396(10258):1204-1222.

23. Instituto de Pesquisa Econômica Aplicada (IPEA), Fórum Brasileiro de Segurança Pública 2019. Atlas da violência 2019. Brasília: Rio de Janeiro: São Paulo: Instituto de Pesquisa Econômica Aplicada; Fórum Brasileiro de Segurança Pública; 2019.

24. Soares Filho AM, Duarte EC, Merchan-Hamann E. Tendência e distribuição da taxa de mortalidade por homicídios segundo porte populacional dos municípios do Brasil, 2000 e 2015. Cien Saude Colet 2020; 25(3):1147-1156

25. Reichenheim ME, Souza ER, Moraes CL, Mello-Jorge MHP, Silva CMFP, Minayo MCS. Violence and injuries in Brazil: the effect, progress made, and challenges ahead. Lancet 2011; 377(9781):1962-1975.

26. Malta DC, Minayo MCS, Soares Filho AM, Silva MMA, Montenegro MMS, Ladeira RM, Morais Neto OL, Melo AP, Mooney M, Naghavi M. Mortalidade e anos de vida perdidos por violências interpessoais e autoprovocadas no Brasil e Estados: análise das estimativas do Estudo Carga Global de Doença, 1990 e 2015. Rev Bras Epidemiol 2017; 20(Supl. 1):[about 14 p.].

27. Organização das Nações Unidas Brasil. ONU: Taxa de feminicídios no Brasil é quinta maior do mundo; diretrizes nacionais buscam solução [Internet]. $2016 \mathrm{Abr}$ [acessado $2017 \mathrm{Fev}$ 1]. Disponível em: https://brasil. un.org/pt-br/72703-onu-taxa-de-feminicidios-no -brasil-e-quinta-maior-do-mundo-diretrizes-nacionais-buscam.

28. Silva MA, Cabral Filho JE, Amorim MMR, Falbo Neto GH. Mulheres vítimas de homicídio em Recife, Pernambuco, Brasil, 2009/2010: um estudo descritivo. Cad Saude Publica 2013; 29(2):391-396.

29. World Health Organization (WHO) [Internet]. Road traffic injuries. Geneva: WHO; 2020 [cited $2021 \mathrm{Feb}$ 09]. Available from: https://www.who.int/news-room/ fact-sheets/detail/road-traffic=-injuries\#: :textApproximately\%201.35\%20million\%20people $\% 20$ die,road $\% 20$ traffic $\% 20$ crashes $\% 20$ by $\% 202020$. 
30. Mascarenhas MDM, Souto RMCV, Malta DC, Silva MMA, Lima CM, Montenegro MMS. Características de motociclistas envolvidos em acidentes de transporte atendidos em serviços públicos de urgência e emergência. Cien Saude Colet 2016; 21(12):3661-3671.

31. Morais Neto OL, Silva MMA, Lima CM, Malta DC, Silva Jr. JB, Grupo Técnico de Parceiros do Projeto Vida no Trânsito. Projeto Vida no Trânsito: avaliação das ações em cinco capitais brasileiras, 2011-2012. Epidemiol Serv Saude 2013; 22(3):373-382.

32. World Health Organization (WHO). Global status report on road safety 2018. Geneva: WHO; 2018.

33. Baldessarini RJ. Epidemiology of suicide: recent developments. Epidemiology and Psychiatric Sciences. [Online] Cambridge University Press; 2020; 29:e71. Available from: doi:10.1017/S2045796019000672

34. Evans E, Hawton K, Rodham K, Deeks J. The prevalence of suicidal phenomena in adolescents: a systematic review of population-based studies. Suicide Life Threat Behav [serial on the Internet]; 2005 Jun [cited 2020 Dez 9]; 35(3):[about 11 p.]. Available from: https://onlinelibrary.wiley.com/doi/abs/10.1521/ suli.2005.35.3.239.

35. Baggio L, Palazzo LS, Aerts DRGC. Planejamento suicida entre adolescentes escolares: prevalência e fatores associados. Cad Saude Publica [periódico na Internet] 2009 Jan [acessado 2020 dez 9]; 25(1):[cerca de 8 p.]. Disponível em: https://www.scielo.br/scielo.php?script =sci_arttext\&pid=S0102-311X2009000100015.

36. Patel V, Saxena S, Lund C, Thornicroft G, Baingana F, Bolton P, et al. The Lancet Commission on global mental health and sustainable development. Lancet [serial on the Internet]. 2018 Out [cited 2020 Dez 9]; 392(10157):[about 45 p.]. Available from: https:// www.thelancet.com/journals/lancet/article/PIIS0140-6736(18)31612-X/fulltext.

37. Felisbino-Mendes MS, Paula TF, Machado ÍE, Oliveira-Campos M, Malta DC. Análise dos indicadores de saúde sexual e reprodutiva de adolescentes brasileiros, 2009, 2012 e 2015. Rev Bras Epidemiol [periódico na Internet]. 2018 Nov [acessado 2020 Dez 9]; 21(Supl. 1):e180013. Disponível em: https:// www.scielo.br/scielo.php?script=sci_arttext\&pid=S1415-790X2018000200415.

38. Brasil. Ministério da Saúde (MS), Secretaria de Vigilância em Saúde. Boletim SINAN, 2019 - AIDS - Boletim Epidemiológico: HIV/Aids - 2019. Brasília: MS; 2019.
39. Reis AAC, Malta DC, Furtado LAC. Desafios para as políticas públicas voltadas à adolescência e juventude a partir da Pesquisa Nacional de Saúde do Escolar (PeNSE). Cien Saude Colet 2018; 23(9):2879-2890.

40. Brasil. Ministério da Saúde (MS). Secretaria de Vigilância em Saúde. Departamento de e Análise de Situação em Saúde. Saúde Brasil 2012: uma análise da situação de saúde e dos 40 anos do Programa Nacional de Imunizações [Internet]. Brasília: MS; 2014.

41. World Health Organization (WHO). Global action plan for the prevention and control of noncommunicable diseases 2013-2020. Genebra [Internet]. 2013 [cited Jan 19 2020]. Available from: https://apps.who.int/ iris/bitstream/handle/10665/94384/9789241506236_ eng.pdf?sequence $=1$

42. Ward JL, Viner RM. The impact of income inequality and national wealth on child and adolescent mortality in low and middle-income countries. BMC Public Health 2017; 17(1):429.

43. Fundo de Emergência Internacional das Nações Unidas para a Infância (Unicef) Brasil. Objetivos de Desenvolvimento Sustentável: Ainda é possível mudar 2030. Unicef Brasil [acessado 2020 dez 9]. Disponível em: https://www.unicef.org/brazil/objetivos-de-desenvolvimento-sustentavel

Artigo apresentado em 02/06/2021

Aprovado em 03/06/2021

Versão final apresentada em 05/06/2021

Editores-chefes: Romeu Gomes, Antônio Augusto Moura da Silva 